\title{
A zebrafish model of Granulin deficiency reveals essential roles in myeloid cell
}

\section{differentiation}

\author{
Clyde A. Campbell ${ }^{1}$, Oksana Fursova ${ }^{1},{\text { Xiaoyi } \text { Cheng }^{1} \text {, Elizabeth Snella }}^{1}$, Abbigail McCune ${ }^{1}$, \\ Liangdao $\mathrm{Li}^{2}$, Barbara Solchenberger ${ }^{3}$, Bettina $\mathrm{Schmid}^{3}$, Debashis Sahoo ${ }^{4}$, Mark Morton ${ }^{5}$, David \\ Traver $^{2,6}$, and Raquel Espín-Palazón ${ }^{1,2,6}$
}

Contact information: espin@iastate.edu; $\underline{\text { dtraver@ucsd.edu }}$

${ }^{1}$ Department of Genetics, Development and Cell Biology. Iowa State University, 2213 Pammel

Drive, Advance Teaching and Research Building 3003, Ames, IA, 50011, USA.

${ }^{2}$ Section of Cell and Developmental Biology. University of California at San Diego, 9500

Gilman Drive, Natural Sciences Building 6107, La Jolla, CA, 92093, USA.

${ }^{3}$ German Center for Neurodegenerative Diseases (DZNE), Munich, Germany

${ }^{4}$ Department of Computer Science and Engineering. University of California at San Diego, La Jolla, CA, 92093, USA.

${ }^{5}$ College of Veterinary Medicine. Iowa State University, IA, USA.

${ }^{6}$ Corresponding authors. 


\begin{abstract}
Granulin $(G R N)$ is a pleiotropic protein involved in inflammation, wound healing, neurodegenerative disease, and tumorigenesis. These roles in human health have prompted research efforts to utilize Granulin in the treatment of rheumatoid arthritis, frontotemporal dementia, and to enhance wound healing. How granulin contributes to each of these diverse biological functions, however, remains largely unknown. Here, we have uncovered a new role for granulin during myeloid cell differentiation. Using a zebrafish model of granulin deficiency, we reveal that in the absence of granulin a (grna), myeloid progenitors are unable to terminally differentiate into neutrophils and macrophages during normal and emergency myelopoiesis. In addition, macrophages fail to recruit to the wound, resulting in abnormal healing. Our CUT\&RUN experiments identify Pu.1, which together with Irf8 positively regulate grna expression. Importantly, we demonstrate functional conservation between the mammalian granulin and the zebrafish orthologue grna. Our findings uncover a previously unrecognized role for granulin during myeloid cell differentiation, opening a new field of study that has the potential to impact different aspects of the human health.
\end{abstract}




\section{Introduction}

Neutrophils and macrophages differentiate from myeloid progenitors and are essential for clearing infections and promoting tissue repair. In addition, recent studies have elucidated a multitude of other functions beyond their classical inflammatory roles. For instance, we and others have demonstrated that neutrophils and macrophages are critical during hematopoietic stem cell (HSC) specification (Espin-Palazon et al, 2014; He et al, 2015; Li et al, 2014; Theodore et al, 2017; Travnickova et al, 2015). Numerous studies have shown that microglia, the tissue resident macrophages of the brain, are involved in neurodegenerative disease (Bachiller et al, 2018; Wynn et al, 2013). In addition, tumor-associated macrophages (TAMs) and neutrophils (TANs) can be key in contributing to tumor metastasis. The tremendous implications that neutrophils and macrophages have in tissue homeostasis and how their disruption leads to human disease have put these cells in the spotlight of many recent investigations. The identification of new molecular regulators of myeloid cell differentiation thus has the potential to broadly impact human health.

Granulin $(G R N)$ is a protein of pleiotropic function that contains several cysteine-rich motifs unique to this molecule (Bhandari et al, 1992). It was first isolated from leukocytes (Bateman et $a l, 1990)$, and It is known to regulate inflammation, wound healing, tissue growth, and it is involved in neurodegenerative diseases, lipofuscinosis, and tumorigenesis (Bateman et al, 2018). Although its cognate receptor has not been identified, granulin can bind to a wide variety of receptors, including TNF receptors, Ephrin type-A receptor 2 (EphA2), Notch, Toll-like receptor 9 (TLR9), LDL Receptor Related Protein 1 (LRP1), and sortilin1 (Chitramuthu et al, 2017). Consequently, Granulin can modulate diverse signaling pathways such as NFkB, WNT, 
MAPK/ERK, PI3K/Akt, and FAK (Alquezar et al, 2016; de la Encarnacion et al, 2016; He et al, 2002; Hwang et al, 2013; Lu \& Serrero, 2001; Tian et al, 2016; Zanocco-Marani et al, 1999).

Many studies support a critical role for granulin during infection and inflammation. While granulin can function as an anti-inflammatory factor, it can also exert a pro-inflammatory role. GRN knockout mice fail to clear Listeria monocytogenes, and their macrophages express high levels of pro-inflammatory cytokines. However, it is surprising that only a few macrophages were found in infected organs (Yin et al, 2010). Moreover, Granulin functions as an antagonist of TNF signaling, playing a critical role in the pathogenesis of inflammatory diseases (Zhu et al, 2002), and has been reported as a promising therapeutic target for inflammatory diseases including rheumatoid arthritis, psoriasis, and osteoarthritis (Farag et al, 2019; Liu, 2011; Tang et al, 2011; Wei et al, 2017).

Several studies have demonstrated that Granulin facilitates wound healing (for review, see Jian and colleagues (Jian et al, 2013)). Administration of GRN to fresh wounds increased the cell counts of neutrophils, macrophages, and fibroblasts, which collectively facilitated healing (He et al, 2003). In addition, it has been shown that macrophages produce granulin as a key regulatory factor in the processes of inflammation and wound healing (Yin et al., 2010).

Mammals encode a single granulin gene $(G R N)$, expressed in most tissues (Bhandari et al, 1993; Daniel et al, 2000). Although recent work indicates that granulin regulates the formation of regulatory T lymphocytes (Kwack \& Lee, 2017; Wei et al, 2014), human hematopoietic cells from the myeloid lineage contain the highest levels of granulin transcripts, being one of the most abundant transcripts in macrophages (Chantry et al, 1998) and monocyte-derived dendritic cells (Hashimoto et al, 1999). Although previous work showed a positive correlation between myeloid 
cell maturation and $G R N$ expression (Ong et al, 2006), a potential role for $G R N$ in myelopoiesis in vivo has not been reported.

Zebrafish (Danio rerio) possess many features that make for an ideal model of embryonic and adult hematopoiesis (Boatman et al, 2013; de Jong \& Zon, 2005; Paik \& Zon, 2010; Stachura \& Traver, 2011; Traver et al, 2004), including high conservation with the human hematopoietic system, external fertilization, high fecundity, rapid development, and embryonic transparency. A whole genome duplication event in teleosts following evolutionary separation from the mammalian lineage resulted in two zebrafish granulin orthologues: granulin a, grna, and granulin b, grnb (Cadieux et al, 2005). We have taken advantage of the tissue-specific segregation of the zebrafish granulin paralogues to assess the functional role of Granulin in hematopoiesis without perturbing other tissues. We demonstrate that whereas grnb is widely expressed in most tissues, grna expression is restricted to myeloid cells. Loss of function experiments using specific morpholinos or grna and grnb null mutants, in combination with in vivo imaging and intracellular flow cytometry, demonstrate that grna, but not grnb, deficiency leads to loss of neutrophils and macrophages due to failure in differentiation from myeloid progenitors during embryonic development. In addition, examination of adult grna mutant zebrafish show failure in neutrophil differentiation in the adult hematopoietic system. Mechanistically, we have performed Cleavage Under Targets and Release Using Nuclease (CUT \& RUN) and show that the master transcription factor of myeloid differentiation Pu.1 directly binds granulin enhancers, triggering its expression. Moreover, our studies demonstrate that Irf8, one of the main transcription factors regulating macrophage development, also acts upstream of granulin. The regulation of mammalian $G R N$ by PU.1 and IRF8 was confirmed using empiric-based databases, highlighting functional conservation between species in myeloid differentiation. Our functional studies in vivo 
demonstrate that loss of grna leads to defective recruitment of myeloid cells to wounds due to a lack of mature macrophages and neutrophils. This results in aberrant collagen deposition within the scar and therefore defective wound healing. Altogether, using our zebrafish model of granulin deficiency, we have discovered that granulin is an essential regulator of myeloid cell differentiation. This study therefore opens a new field of investigation that will help shed light on the pleiotropic functions of this enigmatic protein and facilitate its use as a therapeutic target. 


\section{Results}

\section{grna expression is restricted to myeloid cells in the zebrafish embryo}

Despite mammalian granulin mRNA being among the most abundant transcripts in human macrophages and other myeloid cell lineages (Ong et al., 2006), functional studies on its potential roles in vivo have not been reported. This prompted us to identify an animal model of granulin deficiency amenable to determine the role of granulin during myelopoiesis. Previous work has suggested that whereas grnb is ubiquitously expressed throughout the zebrafish embryo, grna is restricted to the sites of embryonic hematopoiesis (Cadieux et al., 2005).

We aimed to further determine when and where grna and its paralogue grnb were expressed during zebrafish development. First, we performed quantitative PCR (qPCR) in zebrafish embryos from 2-72 hpf. As shown in Figure 1A-B, both grna and grnb transcripts were maternally transferred, since they were detected at 2 hpf, with grna expression 100-200 fold less abundant than grnb (notice different Y axis scales). Zygotic grna and grnb transcripts (detected from 9hpf) were 10 times less abundant than maternally transferred grna and grnb, but were detected throughout all embryonic stages evaluated, with a peak in expression at 72 hpf (Figure 1A-B).

To evaluate where in the embryo grna and grnb were expressed, we generated full-length antisense and sense probes for each granulin paralogue and performed whole mount in situ hybridization (WISH). grna and grnb were expressed by all cells in the early zygote (2-10 hpf) (Supplementary Figure 1). However, grna transcripts were restricted to the embryonic hematopoietic areas in the zebrafish at later developmental stages (48 hpf), including the dorsal aorta (DA) region, and caudal hematopoietic tissue (CHT) (Figure 1C, left and right upper panels 
respectively, black arrowheads). In contrast, grnb was detected throughout the embryo (Figure 1C, lower panels). To confirm the cellular origin of grna expression throughout embryonic development, we utilized SPRING, a tool for interactive exploration of single-cell data from zebrafish embryos (Wagner et al, 2018). Figure 1D-D' shows a single-cell graph of all individual cells across all time points: from $4 \mathrm{hpf}$, center; to $24 \mathrm{hpf}$, outer dots. Cells that expressed grna (green dots) are shown in Figure 1D, and magnified view in 1D'. grna expression was therefore restricted to germline cells (green box) in the early zygote and leukocytes (orange box) at $24 \mathrm{hpf}$, further validating our WISH results. In contrast, grnb was found to be expressed at low levels in all tissues (data not shown).

We next validated the restricted tissue specificity of grna in the late embryo (36-48 hpf) by qPCR of FACS-sorted hematopoietic and non-hematopoietic cells. grna was highly expressed by myeloid cells and absent in non-hematopoietic cells such as muscle and endothelial cells (Figure 1E). Among the myeloid cells expressing grna, macrophages expressed five times more grna transcripts than neutrophils, and 50 times more than developing erythrocytes (Figure 2E).

Altogether, these results demonstrate that in zebrafish, grna expression is restricted to the myeloid cell lineage from $24 \mathrm{hpf}$, while grnb is ubiquitously expressed throughout the embryo.

\section{Grna is required for proper myeloid development}

Due to the restricted expression of grna to developing myeloid cells in the embryo, we hypothesized that grna could be essential for proper myeloid differentiation. To test our hypothesis, we first performed loss-of-function experiments for grna and grnb utilizing specific antisense morpholinos (MOs). In the zebrafish embryo, myeloid progenitors, macrophages, and neutrophils can be visualized as discrete cells by expression of pu.1, mfap4, and mpx, 
respectively, using WISH or the zebrafish transgenes Tg(pU1:Gal4; UAS:eGFP), $T g($ Mpeg 1:eGFP), and $T g(L y z: D s r e d)$, respectively. Grna knock-down utilizing a previously validated MO ( $\mathrm{Li}$ et al, 2010) (Supplementary Figure 2A) significantly reduced in vivo macrophage (mfap4) and neutrophil ( $m p x)$ numbers at 48 hpf compared to grna mismatch control MO injected embryos (Figure 2A upper and middle panels). To investigate if the absence of Grnb also led to myeloid defects, we generated and validated by RT-PCR a specific grnb splice-blocking MO (Supplementary Figure 2C-D). Although some WT grnb transcripts were observed after Grnb-MO injection (Supplementary Figure 2C, lower band), this amount was significantly reduced compared to WT control embryos. No significant changes in neutrophil or macrophage numbers were observed after grnb knock-down (Figure 2A, bottom panel). We further confirmed the loss of macrophages and neutrophils after grna knockdown by using an additional grna MO (grna-MO2) (Li et al., 2010) and performing flow cytometry in pooled Mpeg1.1:eGFP 36hpf embryos (Supplementary Figure 2D-E). As expected, macrophage numbers were significantly reduced in embryos injected with either Grna MO1 or MO2 morpholinos compared to control mismatch MO injected embryos.

To verify that the loss of macrophages and neutrophils was not due to potential off-target effects caused by the grna morpholinos, we generated grna null mutants (Solchenberger et al, 2015) and performed WISH for pu.1, mfap4 and mpx at 48hpf. While myeloid progenitor $(p u .1+)$ numbers were similar (Figures 2B-B''), macrophage and neutrophil numbers were significantly decreased in grna $^{-/-}$compared to grna $^{+/+}$control embryos (Figures 2C-E''). We next performed intracellular flow for Mfap4 to further validate the decrease in macrophages. As expected, the number of Mfap4 $4^{+}$cells was significantly decreased (5-fold) in grna $^{-/}$at $48 \mathrm{hpf}$ (Supplementary 
Figure 2F-G). To ensure that the myeloid defects observed in the absence of grna were not due to a decreased proliferation of differentiated myeloid cells, we performed whole-mount immunohistochemistry (WIHC) for phospho-histoneH3 (P-H3) in 30 hpf Mpx:eGFP transgenic animals and found no colocalization of Mpx:eGFP with $\mathrm{P}-\mathrm{H} 3$ in $g r n a^{+/+}$embryos, indicating that neutrophils do not proliferate during early embryonic development (Supplementary Figure 2H). Altogether, these experiments demonstrate that grna, but not grnb, is essential for the differentiation of myeloid progenitors into macrophages and neutrophils.

\section{Absence of grna leads to long lasting myelopoiesis defects}

We next sought to investigate if grna was also essential for adult myelopoiesis or, in contrast, if these defects were specific to embryonic development. First, we investigated if grna expression was restricted to the myeloid lineage in adult vertebrates. To address this question, we took advantage of the interactive scRNAseq data published by Tang and colleagues (Tang et al, 2017) from adult wildtype zebrafish kidneys, the hematopoietic zebrafish analogue to mammalian bone marrow. grna expression was restricted to the cell clusters defined as macrophages (yellow circles) and myeloid cells (green circle) (Figure 3A and Supplementary Figure 3A). In contrast, grnb transcripts were present at low levels in most hematopoietic cells, but highly enriched in multicilliated cells and vascular endothelium (Sup Figure 3A), which are non-hematopoietic cells within the kidney. We next wanted to address if, similar to the zebrafish orthologue grna, mammalian granulin expression was also up-regulated in myeloid cells. First, we utilized Gene Expression Commons (Seita et al, 2012) to query the dynamic-range of granulin within microarrays of the mouse (Mus musculus) hematopoetic system (https://gexc.riken.jp/models/3/genes/Grn?q=Grn). As shown in Figure 3B, Grn expression was active in hematopoietic stem cells (HSCs), up-regulated in granulocyte/macrophage progenitors 
(GMPs) and reached its highest expression in granulocytes (Gra) and monocytes (Mono). In contrast, cells of the megakaryocyte/erythrocyte lineage, including megakaryocyte/erythrocyte progenitors (MEPs), Megakaryocyte progenitors (Mkps), and Colony Forming Unit-Erythroid (PCFU-e) drastically down-regulated Grn expression. Second, to confirm these results at the single cell level, we utilized the hematopoietic single cell interactive gene viewer based on mouse sorted hematopoietic cells published by Olsson and colleagues (Olsson et al, 2016) and found that murine $G R N$ is highly expressed in myeloid cells including monocytes, granulocytes, and myelocytes, mimicking the expression of the master myeloid transcription factor PU.1 (Supplementary Figure 3B, black squares). Both Grn and Pu.l were down-regulated in cells of the erythroid lineage (Supplementary Figure 3B, purple squares). Altogether, these data show conserved expression for the mammalian granulin and the zebrafish orthologue grna in hematopoietic lineages.

To assess the effect of grna loss on myeloid cell differentiation in the adult vertebrate, we collected whole kidney marrow cells from $\mathrm{grna}^{-/-}$and control $\mathrm{grna}^{+/+}$siblings and performed flow cytometry to quantify myeloid cell numbers using the forward and side scatters as we described previously (Traver et al, 2003). The percentage of myeloid cells within the kidney marrow of $\mathrm{grna}^{-/-}$was significantly decreased as compared to $\mathrm{grna}^{+/+}$control kidneys (Figure 3C and Supplementary Figure 3C). We next cytospun grna $^{-/-}$and $g r n a^{+/+}$control sibling kidney cell suspensions and subsequently performed Wright-Giemsa staining to examine hematopoietic counts and morphology. Pathologist examination revealed that $\operatorname{grna}^{-/-}$had increased early myeloid precursors (Figure 3D, orange arrowheads) with decreased differentiation into mature neutrophils (green arrowheads) based on 200 non-erythroid nucleated differential cell counts (Figure 3D-E). Importantly, there were no differences in body size between $\mathrm{grna}^{+/+}$and $\mathrm{grna}^{-/-}$ 
sibling fish as measured by length from mouth to caudal fin (Supplementary Figure 3D). Taken together, these data indicate that grna is essential to drive myeloid cell differentiation from early myeloid precursors in adult zebrafish.

\section{grna inhibits erythroid differentiation}

Next, we performed bulk RNA-seq in kidney marrow from adult $\mathrm{grna}^{-/-}$and $\mathrm{grna}^{+/+}$control siblings to identify genes whose expression was dysregulated in the absence of grna. We found 154 genes significantly down-regulated in the grna mutants (Table S2), and 116 genes significantly up-regulated (Figure 4A-C) (Table S3). As expected, we found important myelopoiesis-related genes down-regulated in grna mutants, including cebpa, rgs 2 , apoeb and abcalb (Figure 4B-C). Interestingly, when we looked at the cell types that expressed these down-regulated genes using the online scRNA-seq viewer from zebrafish kidney marrow (Tang et al., 2017), we found that almost half of them (64 out of 154) (Table S4) were restricted to myeloid cell subpopulations (Sup Figure 4), supporting a role for grna in myeloid cell differentiation. In addition, many genes down-regulated in the grna mutants have been shown to participate in inflammation and immune response, including ill3ral, il4r.1, irf2a, itgb3a, nfkbie, $c p$, tlr5b, cxcl12a, hbegfa, $m l x$ and atg16l1 (Figure 4B-C and Sup Fig 4). Altogether, this data further confirms that grna is essential for proper myeloid cell differentiation.

Unexpectedly, among the up-regulated genes in $g r n a^{-/}$, we found genes described to be important for red blood cell development (alas2), erythrocyte shape (epb41b), hemoglobin transport (hbae4), as well as platelet adhesion (gplbb) (Figure 4B-C). Surprisingly, the expression of 27 out of 116 upregulated genes was found to be restricted to erythrocytes and, to a lesser extent, platelets (Sup Figure 4 and Table S5). We then hypothesized that grna might inhibit erythroid differentiation. To address this, we injected Grna-MO and Grna mismatch 
control MO into gatala:DsRed reporter zebrafish embryos. Gata1 is a master transcription factor that drives erythroid cell differentiation. We found a significant increase in Dsred expression in Grna morphants compared to control siblings (Figure 4D), suggesting that grna inhibits gatala. We next quantified this up-regulation using flow cytometry and found a 10-fold increase in DsRed intensity in the absence of grna (Figure 4E), indicating that the gatala promoter is 10 times less active in vivo in the absence of Grna. Altogether, these results show that Granulin inhibits erythroid differentiation and facilitates myeloid cell development in vivo.

\section{Pu.1 and Irf8 control grna expression in zebrafish, and this regulatory mechanism is conserved for mammalian GRN}

Pu.1, encoded by the Sfpil (Spi-1) gene, is a master transcription factor that leads to myeloid cell specification. Pu.1 contains various Ets domains that recognize the DNA sequence harboring the core GGAA motif (Burda et al, 2010) (Figure 5D). Since pu.1 expression was unaltered in the absence of grna (Figures 2B-B'), we hypothesized that grna acted downstream of Pu.1 for myeloid cell specification. First, to address if grna and pu.l transcripts were co-expressed by the same cells, we performed double fluorescence in situ hybridization (FISH) for grna and pu.l in 48 hpf embryos. Figure 5A shows co-localization of grna and pu.1 at a single cell resolution using confocal microscopy. To demonstrate that Pu.1 genetically acts upstream of grna, we utilized a specific MO against Pu.1 (Rhodes et al, 2005) and performed WISH for grna and the neutrophilic marker mpx. As shown in Figure 5B (left and middle panels), Pu.1 knockdown completely abolished grna expression, as well as decreased the neutrophilic marker mpx as previously reported (Espin-Palazon et al., 2014). To control for the efficiency of the Pu.1-MO, we injected Mpeg1:eGFP embryos and found that macrophages were completed ablated in the absence of Pu.1 as expected (Supplementary Figure 5A). To address if Pu.1 directly bound grna 
enhancers to promote its transcription, we utilized the motif comparison tool Tomtom (http://meme-suite.org/tools/tomtom) (Bailey et al, 2009) to identify putative Pu.1 binding sites in grna regulatory sequences. Using the PU.1 matrix ID: MA0080.5 http://jaspar.genereg.net/matrix/MA0080.5/ from Homo sapiens derived from ChIP-seq (Chromatin immunoprecipitation followed by sequencing) data (Ray-Gallet et al, 1995) (Figure 5D), we found several putative binding sites (BS) in the non-coding regions flanking the grna gene (Figure 5C). These included three predicted BS in grna intron 3-4 (BS1 at +5655 , BS6 at +4485); and three in the grna 5' enhancer region (BS2 at -6935 , BS5 at -7843, BS3 at -14045) (Figure 5C). We then dissected zebrafish kidney marrow to perform Cleavage Under Targets and Release Using Nuclease (CUT\&RUN) with a specific antibody for zebrafish Pu.1 followed by qPCR to amplify each predicted pU.1 BS. We chose CUT\&RUN as a chromatin profiling strategy since it is robust and has extremely low background signal (Skene \& Henikoff, 2017). While putative $\mathrm{Pu} .1 \mathrm{BSs} 5$, and 6 showed no enrichment compared to control isotype $\operatorname{IgG}$ antibody, Pu.1 BS1, BS2 and BS3 showed two- to eight-fold enrichment (Figure 5E), demonstrating that Pu.1 directly binds grna regulatory sequences. To gain further insight into the grna regulatory gene network, we knocked down Irf8, an essential transcription factor required for macrophage specification. It has been demonstrated that Pu.1 acts upstream of Irf8, and that both transcription factors cooperate to regulate granulocyte-macrophage fate decisions in myeloid progenitors and maturation of macrophage precursors in both zebrafish and mice (Kurotaki et al, 2018; Li et al, 2011; Shiau et al, 2015).

Irf8 depletion by utilizing a specific Irf8 morpholino (Li et al., 2011) led to increased $m p x$ expression (Figure 5B, right top panel) and loss of macrophages (Supplementary Figure 5A) as previously reported (Espin-Palazon et al., 2014). In addition, Irf8 knockdown resulted in a 
significant decrease in grna expression (Figure 5B, right bottom panel). Using Gene Expression Commons (Seita et al., 2012) to query the dynamic-range of Irf8 expression within microarrays of the mouse (Mus musculus) hematopoietic system, we found that Irf8 is up-regulated in myeloid precursors (Supplementary Figure 5B). Together, these results indicate that Irf8 acts genetically upstream of grna to trigger grna expression within myeloid precursors.

We next wanted to investigate if the transcriptional network that regulated grna in zebrafish myeloid cells was also conserved in mammals. We queried if PU.1 bound the human GRN promoter by using the regulatory feature of ensembl.org (Zerbino et al, 2016). This database uses a variety of published genome-wide assays, such as ChIP-seq data on chromatin structure, epigenomic, and transcription factor binding to identify peaks in the genome that correspond to binding of a TF. We found that in human cells, PU.1 bound to the first intron of the human GRN gene (Supplementary Figure 5C). Next, we utilized ChIP Enrichment Analysis (ChEA) (Lachmann et al, 2010) to search for other TFs binding the mammalian GRN regulatory regions. ChEA utilizes transcription factor ChIP-seq studies extracted from supporting materials of publications to identify peaks at the promoter of a queried gene. We found that the myeloid TFs IRF8 and CEBPB bound the $G R N$ promoter in mouse with significant $\mathrm{P}$-values $<0.05$ (Supplementary Figure 5D). In addition, hematopoietic and inflammatory TFs including RUNX1, Growth Factor Independent 1 Transcriptional Repressor (GF1), Nuclear Factor, Erythroid 2 Like 2 (NFE2L2) and RELA Proto-Oncogene, NF-KB Subunit (RELA) were also detected to bind mammalian GRN, although with lower significance (Supplementary Figure 5D). We next used Harmonizome (Rouillard et al, 2016) in combination to ChEA to identify genes that were co-expressed with GRN. As expected, mammalian PU.1 and IRF8 were co-expressed 
with GRN (Supplementary Figure 5E), as well as myeloid specific genes such as MPEG, CD68, and TREM2 with a Pearson Correlation >0.6 (Supplementary Figure 5F).

Taken together, these data demonstrate that in zebrafish, Pu.1 and Irf8 positively control grna expression in myeloid cells, and that this regulatory mechanism is conserved for human and mouse granulin.

\section{grna is required for emergency myelopoiesis and macrophage recruitment to the wound}

Emergency myelopoiesis is the proliferation and differentiation of hematopoietic progenitor cells towards the myeloid lineage as a result of increased demand following injury or infection (Mitroulis et al, 2018). We have shown that myeloid progenitors are present in the absence of grna (Figures 2B-B' and 4F-G). Because grna is required for normal myelopoiesis, we hypothesized that the absence of grna would lead to an aberrant emergency myelopoiesis response. To investigate if myeloid progenitors could respond to emergency myelopoiesis, transgenic Mpeg1:eGFP embryos were injected with Grna or Grna control mismatch MOs and caudal tails were resected at 48 hpf to trigger an emergency myelopoiesis response. Fluorescent images were taken from 1 hour-post wounding (hpw) to $32 \mathrm{hpw}(80 \mathrm{hpf})$, and macrophage numbers were quantified in the tail (CHT and wound) (Figure 6A). While macrophage numbers increased in all individuals injected with Grna mismatch control morpholino at $9 \mathrm{hpw}$, Grna morphants failed to generate more macrophages (Figure 6B), suggesting that Grna is required for emergency myelopoiesis. Although the absence of grna led to a remarkable decrease in macrophage number, a small percentage of macrophages was still present in grna mutants and morphants (Figure 2). Therefore, we sought to determine if these macrophages were able to respond and recruit to the injury site using the same experimental approach described above (Figure 6A). As expected, the number of macrophages recruited to the wound was significantly 
decreased in the absence of Grna (Figure 6D). This could be due to the reduced numbers of macrophages in the Grna morphants, but the percentage of recruited macrophages with respect the total macrophage number per individual could be normal. To address this, we quantified the number of macrophages recruited to the wound and normalized it to the total number of macrophages in the tail (CHT and wound) for each individual. The percentage of macrophages that were recruited to the wound with respect to the total macrophage numbers was significantly reduced in the absence of Grna (Figure 6C) compared to control Grna mismatch morpholino injected siblings. Taken together, these results indicate that Grna is essential for emergency myelopoiesis and that the macrophages produced in the absence of Grna have functional abnormalities, not being able to properly respond to tissue injury.

\section{Wound healing is impaired in grna mutants}

It has been recently demonstrated that macrophages are one of the main contributors to tissue repair in both mammals and zebrafish (Li et al, 2012; Minutti et al, 2017; Miskolci et al, 2019; Morales \& Allende, 2019; Nguyen-Chi et al, 2017; Simoes et al, 2020). They contribute directly and indirectly by ingesting apoptotic neutrophils, triggering the resolution of inflammation, breaking down the injured matrix, re-epithelialization, vascularization of the wound bed, releasing growth factors, induction of extracellular matrix deposition (Minutti et al., 2017), and even depositing collagen themselves as shown recently (Simoes et al., 2020). In zebrafish, specific ablation of macrophages severely impedes inflammatory resolution and tissue regeneration (Li et al., 2012). Moreover, it is known that Granulin facilitates wound healing by increasing macrophage, fibroblast, and blood vessel number in the injured tissue (He et al., 2003). Based on these data, we hypothesized that wound healing would be impaired in $\mathrm{grna}^{-/-}$ embryos due to the decreased and abnormal production of macrophages, and that the functions 
attributed to Granulin during wound healing were the consequence of the myelopoietic defects described herein (Figures 2-4). To test our hypothesis, we performed tail fin resection at 48 hpf in grna $^{-/-}$and grna $^{+/+}$control embryos and subsequently imaged regenerated tissue over three days (72hpw) (Figure 7A). Tissue regeneration was deeply impaired in grna $^{-/-}$larvae as shown in Figure 7B. First, the area of tissue regenerated was significantly diminished in grna $^{-/-}$compared to $\mathrm{grna}^{+/+}$at all times evaluated (Figure 7C). While grna $^{+/+}$control larvae completely regenerated the tail fin at $72 \mathrm{hpw}$, grna $^{-/}$failed to do so (Figure 7B-C). Second, collagen organization in the regenerated area of $\mathrm{grna}^{-/-}$was deeply disrupted as shown in Figure 7D. While collagen fibers in $\mathrm{grna}^{+/+}$control individuals were perpendicular to the wound edge (Figure 7D, left panel), aligned fibers were disarrayed and less perpendicular to the wound edge in grna $^{-/-}$(Figure 7D, right panel). These observations are consistent with, and extend previous findings that macrophages indirectly and directly support collagen fiber production and organization during wound repair. Altogether, these results link for the first time the role of granulin in wound healing with the myelopoietic defects described here, and validate functional conservation between the mammalian granulin and the zebrafish orthologue grna. Overall, these data also reveal the TFs controlling grna expression and the proteins that act downstream of Grna to facilitate myeloid cell differentiation and inhibit the erythroid program (Figure 7E). 


\section{Discussion}

Granulin profoundly impacts wound healing, autoimmune diseases, tumorigenesis, aging, and lysosome biology. In addition, loss of function mutations in the granulin gene are causative for Frontotemporal Lobar Degeneration (FTD) and Ceroid Lipofuscinosis (Petkau \& Leavitt, 2014; Ward et al, 2017). The tremendous implications that granulin function has in human health, have put this enigmatic protein in the spotlight of many investigations to utilize it as a therapeutic target. Despite its medical importance, why and how granulin can influence these diverse biological processes have remained unclear. Here, we have uncovered a new in vivo function for granulin in myeloid cell differentiation. We revealed that loss of granulin causes less macrophages and neutrophils to differentiate with consequences for wound healing and inflammation.

In contrast to mammalian granulin, which is highly expressed by myeloid cells but also present at lower levels in non-hematopoietic tissues, we have demonstrated that the zebrafish granulin orthologue grna is restricted to hematopoietic cells, while grnb is expressed in most tissues. Our data suggests that grna evolved to specifically take on the hematopoietic functions of the mammalian granulin. The tissue-specific segregation of the zebrafish granulin paralogues has therefore allowed an unprecedented manner of assessing granulin function in hematopoiesis without perturbing other tissues. Using our zebrafish model of grna deficiency, we have demonstrated that grna is essential for neutrophil and macrophage differentiation from myeloid progenitors during normal and emergency myelopoiesis, therefore impacting inflammation and wound repair. Importantly, we show that the regulatory mechanisms of expression of grna and mammalian granulin by hematopoietic cells is highly conserved among zebrafish and mammals. 
In the absence of grna, we persistently observed a profound defect in neutrophil differentiation, while some macrophages still developed. Our data support a functional and transcriptional dysregulation in macrophages in the absence of grna, since: (1) Macrophages failed to recruit to the wound; (2) new macrophages failed to generate during emergency myelopoiesis; and (3), many of the down-regulated genes in the grna $^{-/-}$vs. grna $^{+/+}$kidneys are known anti-inflammatory genes, and some completely restricted to macrophages (abcalb, il4rl). This aberrant production of inflammatory genes in the absence of grna indicates a dysregulated inflammatory response, which is consistent with previous reports in mice lacking Grn (Yin et al., 2010) and our previous data showing morphological and transcriptional changes in microglial cells, the resident macrophages of the brain, in zebrafish lacking grna and grnb, indicative of a pro-inflammatory phenotype (Zambusi et al, 2020). These observations also support functional conservation between the mammalian granulin and the zebrafish orthologue grna.

The transparency of zebrafish larvae allowed us to perform functional assays to visualize the recruitment of macrophages to wounded tissue and its repair. We demonstrate that wound healing is abnormal in individuals lacking grna, as previously reported in granulin knockout mice (He et al., 2003). However, the restricted grna expression within hematopoietic cells in our zebrafish model of grna deficiency allowed us to address that the defects in wound healing are caused, at least mostly, by aberrant myeloid cell differentiation. In accordance to our data in zebrafish, it has been reported that the majority of the granulin expressed in the wound derived from myeloid cells (He et al., 2003). Since macrophages greatly contribute to tissue repair, it is not surprising that impaired myeloid differentiation leads to aberrant healing of the wounded tissue. Altogether, we have been able to reproduce previously described phenotypes of mice 
models of granulin deficiency in our grna zebrafish mutants and address that the cause of aberrant inflammation and wound healing is due to impaired myelopoiesis.

Our work here also opens the outstanding question of whether the contributions described for granulin in tumorigenesis (Arechavaleta-Velasco et al, 2017) are also due to a likely abnormal production of tumor associated neutrophils (TANs), macrophages (TAMs), and antitumor macrophages that constitute the tumor microenvironment, impacting the tumor progression (Pathria et al, 2019). In addition, our discovery here paves the way for the understanding of how granulin leads to frontotemporal dementia. In the last years, multiple lines of evidence reveal the impact of microglia in neurological disorders (Bachiller et al., 2018; Heneka, 2019). The production of aberrant microglia in the absence of granulin has also been described recently in the adult zebrafish (Zambusi et al., 2020) and mice (Bright et al, 2019; Lui et $a l, 2016)$, being the cause rather than the consequence of neurodegeneration. A plausible hypothesis is therefore that microglia-driven neuroinflammation in FTD is caused by the myeloid defects described here in myeloid development. Altogether, our results suggest genetic and functional differences in macrophages produced in the absence of grna, which most likely impact the homeostasis of many tissues due to the ubiquitous presence of resident macrophages and the implications that macrophages and neutrophils have in tumor progression, autoinflammatory disease and neurodegenerative disease. More experiments in each of the contexts will be needed to address the exact differences among microglia or tumor associate myeloid cells in the absence of granulin, and our zebrafish model of granulin deficiency is an ideal model to perform these studies in vivo.

Among the complex regulatory network of TFs that regulate hematopoiesis, GATA1 and PU.1 are key on regulating the erythroid versus myeloid program by antagonizing each other 
(Iwasaki \& Akashi, 2007; Monteiro et al, 2011; Wolff \& Humeniuk, 2013; Zhang et al, 2000). These TFs are therefore main contributors of the pathogenesis of hematopoietic disorders (Burda et al., 2010). Despite decades of efforts trying to address how PU.1 and GATA1 negatively regulate each other, little is still known about this mechanism. In this study, we demonstrate that $\mathrm{Pu} 1$ positively regulates grna expression, and that this regulatory mechanism is highly conserved in the mammalian granulin. Importantly, our results also indicate that granulin inhibits gatal expression in vivo. Our findings identify granulin as a previously unrecognized regulator of the Pu.1/Gata1 antagonism and extend previous results on the mechanism of the gatal inhibition by $\mathrm{Pu}$.1. Further studies will be required to determine the precise molecular mechanism by which granulin inhibits gatal expression. In addition, our first report of the use of CUT\&RUN in zebrafish greatly expands our zebrafish genetics toolkit. Since this technique can be performed in a small number of cells with exceptionally low background and high specific signal (Skene \& Henikoff, 2017), it will facilitate the discovery of new regulatory networks of TFs that affect gene expression in the zebrafish model, a small animal with relatively low numbers of cells.

In conclusion, with our discovery that granulin is essential for normal myelopoiesis, it is not surprising that its dysregulation leads to pleiotropic effects, since macrophages and neutrophils participate in inflammation, wound healing, tumorigenesis, and neurodegeneration. Our work therefore opens a new field of study that has the potential to impact different aspects of the human health, filling a knowledge gap needed to advance the manipulation of GRN as a therapeutic target. Last, our results are expected to advance understanding of how this protein could be manipulated to treat hematopoietic disorders such as neutropenia or myeloid leukemia. 
Since granulin is a secreted factor, it may be plausible to be used as a therapeutic target to treat these hematological disorders, expanding the treatment options for these patients.

\section{Materials and Methods}

\section{Zebrafish husbandry and strains}

WT $\mathrm{AB}^{*}$ and transgenic zebrafish (Danio rerio) embryos and adults were mated, staged, raised and processed as described (Westerfield, 2000) in a circulating aquarium system (Aquaneering) at $28^{\circ} \mathrm{C}$ and maintained in accordance with ISU and UCSD IACUC guidelines. Mutant grna $^{\text {mde54a }}$ (Solchenberger et al., 2015), transgenic Tg(mpx:eGFP) ${ }^{i 114}$ (Renshaw et al, 2006), Tg(gatal:DsRed) $)^{\text {sd2 }}$ (Traver et al., 2003), Tg(mpeg1:eGFP) ${ }^{g l 22}$ (Ellett et al, 2011), $\operatorname{Tg}(k d r l: H s H R A S-m C h e r r y)^{s 896}$ (referred to as $k d r l: m C h e r r y$ throughout manuscript) (Bertrand et al, 2010), $\operatorname{Tg}(\text { Lyz:Dsred2) })^{n z 50}$ (Hall et al, 2007), Tg(Myf5:eGFP) $)^{z f 42}$ (Chen et al, 2007), $\operatorname{Tg}\left(\right.$ LCR:eGFP) ${ }^{\mathrm{cz} 3325}$ (Ganis et al, 2012) and various intercrosses of these lines were utilized.

\section{Morpholino injection}

Specific antisense morpholinos (MOs) (Gene Tools) were resuspended in water at 2mM. MOs used in this study were Standard-MO (Gene Tools), pU1-MO (Rhodes et al., 2005), irf8-MO (Li et al., 2011), Grna-MO1 (also denoted as Grna-MO throughout the manuscript) 5'TTGAGCAGGTGGATTTGTGAACAGC-3' (Li et al., 2010), Grna control morpholino with 5

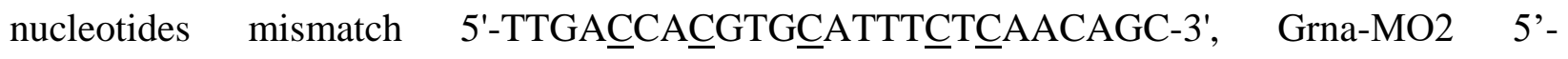
GGAAAGTAAATGATCAGTCCGTGGA-3' (Li et al., 2010), and Grnb-MO 5'CCACAGCGCAACTCTCACACCTG-3' (validated in this manuscript). Morpholinos were diluted in water at a concentration of $0.4 \mathrm{mM}$ (Grna-MO), $0.4 \mathrm{mM}$ (Grna mismatch-MO), 0.2 mM (Grna-MO2), $0.6 \mathrm{mM}$ (Grnb-MO), $1.4 \mathrm{mM}$ (irf8-MO) and $2 \mathrm{mM}$ (pu1-MO) with phenol red 
solution and $2 \mathrm{nl}$ were injected into the yolk ball of one-cell-stage embryos using a micromanipulator (Narishige) and PM 1000 cell microinjector (MDI). For Grnb-MO validation, 2024 hpf zebrafish embryos injected with Grnb-MO or uninjected controls were collected and RNA was isolated with RNeasy (Qiagen), cDNA generated with qScript Supermix (Quanta BioSciences) and PCR performed with primers Grnb-F2-MO and Grnb-R2-MO (Table S1) for the detection of the wildtype (498 bp) or the mutant (535 bp) grnb amplicon. These amplicons were sequenced. The mutant grnb amplicon contained 37 extra-nucleotides that changed the reading frame of the grnb mRNA.

\section{Quantitative RT-PCR analysis}

RNA was isolated from tissues with RNeasy (Qiagen), and cDNA generated with qScript Supermix (Quanta BioSciences) or iScrip gDNA Clear cDNA Synthesis Kit (BioRad, 1725035). Primers to detect zebrafish transcripts are described in Table S1. qPCR was performed in CFX real-time PCR detection system (BioRad), and relative expression levels of genes were calculated by the following formula: Relative expression $=2^{-(\mathrm{Ct}[\text { gene of interest }] \text {-Ct[housekeeping gene }])}$.

\section{Flow cytometry}

To quantify the myeloid fraction in kidney marrows from $\mathrm{grna}^{-/-}$and $g r n a^{+/+}$control siblings, adult zebrafish between three to nine months old were anesthetized in tricaine, subjected to cardiocentesis and kidney dissection as previously described (Traver et al., 2003). The resulting kidney suspension was gently triturated with a P1000 pipette and filtered with a $30 \mu \mathrm{m}$ cell strainer (Thermo Fisher Scientific, NC9084441) and stained with Sytox Red (Life Technologies) to exclude dead cells. Flow cytometric acquisitions were performed on a LSR-Fortessa (BD) and analyses were performed using FlowJo software (v10.3, Tree Star) as previously described (Traver et al., 2003). 
To analyze macrophage numbers in Grna morphants, three 48 hpf Mpeg 1:eGFP+ embryos per replicate (three to four replicates per experiment) previously injected with Grna-MO1, GrnaMO2 or Grna mismatch MO were dechorionated with pronase (Roche) and anesthetized in tricaine. Gently triturated with a P1000 pipette and chemically dissociated with liberase TM (Roche) for 20 minutes in agitation. The resulting cell suspension was filtered and stained with Sytox Red to exclude dead cells. Flow cytometric acquisitions were performed on a FACS LSRFortessa (Becton Dickinson) and analyses were performed using FlowJo software (v10.3, Tree Star).

\section{Intracellular flow cytometry}

Flow cytometry was performed on a LSR Fortessa (BD) and data were analyzed using FlowJo

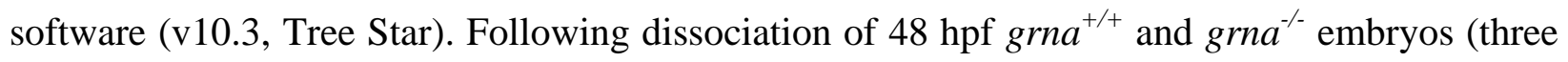
embryos per condition) with liberase TM (Roche) as described below, cells were fixed with PFA $4 \%$ and permeabilized for 30 minutes on ice with PBS containing $0.1 \%$ triton. Intracellular staining was performed with anti zebrafish mfap4 antibody (Gentex, GTX132692), dilution 1:250, followed by staining with $5 \mathrm{ug} / \mathrm{ml}$ of goat anti-rabbit $\operatorname{IgG}(\mathrm{H}+\mathrm{L})$ antibody, alexa-488 (Thermo Fisher, A11034).

\section{Fluorescence-activated cell sorting (FACS)}

To isolate embryonic cells for qPCR analysis, approximately $20016 \mathrm{hpf} M y f 5: e G F P+$; $22 \mathrm{hpf}$ Kdrl1:mCherry+, Gatal:Dsred-; 48 hpf Mpx:eGFP; 48 Mpeg1:eGFP; or 36 hpf LCR:eGFP embryos were dechorionated with pronase (Roche) and anesthetized in tricaine. Gently triturated with a P1000 pipette. The resulting cell suspension was filtered and stained with Sytox Red to 
exclude dead cells. Cell sorting of positive fluorescent cells was performed with a FACS ARIAII (Becton Dickinson).

\section{In Situ Hybridization}

WISH was carried out as described (Thisse et al, 1993). Probes for the grna, grnb, pu.1, mfap4, $m p x$, and apoeb transcripts were generated using the DIG RNA Labeling Kit (Roche Applied Science) from linearized plasmids. Embryos were imaged using a Leica M165C stereomicroscope equipped with a DFC295 color digital camera (Leica) and FireCam software (Leica).

Double fluorescent in situ hybridization (FISH) was performed as previously described (Brend \& Holley, 2009) using the following reagents: TSA Plus Cyanine 3/Fluorescein system (NEL753001KT), anti-Digoxigenin-POD, Fab fragments (11207733910), and anti-fluoresceinPOD, Fab (11426346910). Embryos were analyzed using a Sp5 confocal (Leica).

\section{Whole-mount immunohistochemistry}

Whole-mount immunohistochemistry for immunofluorescence staining of P-Histone H3 Ser10 in 48 hpf $T g(M p x: e G F P)$ zebrafish embryos was performed as previously described (Espin et al, 2013). The following antibodies were used: rabbit anti-phospho-Histone H3 (Ser10) antibody (Millipore, 06-570) (dilution 1:100), anti-GFP antibody chicken IgY (Aves Labs GFP-1020) (dilution 1:500), goat anti-Chicken IgY (H+L) Alexa-488 (Thermofisher A-11039) (dilution 1:500), donkey anti-rabbit IgG (H+L) Alexa-594 (Thermofisher A21207) (dilution 1:500). The samples were imaged with a Leica Sp5 confocal.

\section{Enumeration of myeloid progenitors, macrophages and neutrophils}


Animals were subjected to WISH for pu.1 (myeloid progenitors), mfap4 (macrophages), or mpx (neutrophils) at $48 \mathrm{hpf}$ at varying locations (tail or head, listed in figures) and cells expressing the above mentioned transcripts were imaged and manually counted per individual. To image and enumerate macrophages in vivo after tail resection, fluorescence microscopy was performed on Mpeg1:eGFP transgenic animals. Z-sections of the tail region were captured using Leica Thunder imager with DFC9000 GT camera and LAS X navigator, and manually counted.

\section{Fin amputation surgeries}

48hpf zebrafish embryos were anesthetized with tricaine and a single cut traversing the entire dorsoventral length of the caudal fin was made using a surgical scalpel size \#15 (19-200-218, fisher scientific). Embryos were individually isolated and imaged at each given timepoint. Throughout the time course, the initial amputation plane of each embryo was determined by superimposing the image captured at each timepoint with the image of the initial cut using Adobe Photoshop CS6. The caudal-most tip of notochord was used as a landmark for spatial alignment. Quantification of regenerate area was determined using ImageJ.

\section{Cytology}

Cytospin preparations were made with $1 \times 10^{5}$ to $2 \times 10^{5}$ kidney cells cytocentrifuged at $300 \mathrm{rpm}$ for 5 min onto glass slides in a Thermo Scientific Shandon CytoSpin 4. Cytospin preparations were processed through Wright-Giemsa stains (Fisher Scientific, 5029782) for morphological analyses and differential cell counts. Briefly: Wright Stain Solution was placed upon the smear for 2 mins and washed with distilled water. Subsequently, the slides were placed for 10 minutes in a coplin jar containing Giemsa and rinsed with distilled water.

\section{Morphological analyses and differential cell counts of kidney marrow hematopoietic cells}


200 non-erythroid nucleated differential cell counts were assessed in grna $^{-/-}$or control grna+/+ siblings kidneys after cardiocentesis, kidney dissection, cytospin and staining with WrightGiemsa as described here. The morphological features to identify each cell type are the following. Early myeloid precursors, round to ovoid in shape with a round to ovoid-shaped pink nucleus. Chromatin pattern finely granular and semi frequently with distinct round nucleoli. High nuclei to cytoplasmic $(\mathrm{N}: \mathrm{C})$ ratio with a moderate amount of deep blue cytoplasm. Late myeloid precursors (immature neutrophils): round to ovoid to bilobed nuclei are with light purple to darker pink coloration. Slightly clumped chromatin pattern and no distinct nucleoli. Moderate $\mathrm{N}: \mathrm{C}$ ratio with a blue to light blue cytoplasm. Neutrophils (mature): smaller in size than late myeloid precursors. Round to ovoid to bilobed to segmented dark purple nucleus smaller than late myeloid precursors. Moderate $\mathrm{N}: \mathrm{C}$ ratio with a light blue cytoplasm and often bubbly vacuolated appearance. Macrophages: round to ovoid in shape. Nuclear shapes included round, ovoid, bilobed, and lobulated forms that have a pale pink to purple coloration. The chromatin pattern is finely stippled that occasionally has distinct nucleoli. Moderate $\mathrm{N}: \mathrm{C}$ ratio that is moderately high with a blue appearance frequently vacuolated and sometimes contained melanin pigment. Lymphocytes: small round to ovoid-shaped cells. Round to ovoid-shaped nucleus, coarsely clumped chromatin pattern, and no distinct nucleoli. High N:C ratio with a scant amount of pale blue cytoplasm.

\section{CUT\&RUN}

Adult wild type $\mathrm{AB}^{*}$ zebrafish between three to nine months old were anesthetized in tricaine, subjected to cardiocentesis and kidney dissection as previously described (Traver et al., 2003).

The resulting kidney suspension was gently triturated with a P1000 pipette and filtered with a 30 $\mathrm{m}$ cell strainer (Thermo Fisher Scientific, NC9084441). 90,000 -120,000 cells were used per 
condition to perform CUT\&RUN using CUT\&RUN assay kit (86652S, Cell signaling) with an anti zebrafish Pu.1 antibody (GTX128266, GeneTex) $(4 \mu \mathrm{l})$ or rabbit (DA1E) IgG Isotype control antibody (as recommended in kit protocol) following the manufacturer instructions with the following modifications: Digitonin dilution 5:1000. Incubation time for primary antibody: 10 hours at $+4^{\circ} \mathrm{C}$; for pAGMase: 1 hour at $+4^{\circ} \mathrm{C}$. Digestion time: $30 \mathrm{~min}$.

\section{In silico prediction of Pu.1 binding sites}

To identify potential binding sites for Pu.1 in the promoter and enhancer regions of grna, the entire grna gene sequence plus $10 \mathrm{~Kb} 5^{\prime}$ ' upstream grna were subjected to $\mathrm{Pu} .1$ binding sites prediction using Consite (http://consite.genereg.net/) and Tomtom (http://memesuite.org/tools/tomtom). The transcription factor binding profile matrix for Pu.1 ID: MA0080.4 from JASPAR was utilized when using Tomtom, and user defined profile (http://jaspar.genereg.net/matrix/MA0080.4/) and 70\% identity analyses were used for Consite. The fragment located at +23765 from the grna ATG start codon was used as a control, since it lacked any predicted binding sites for Pu.1.

\section{Preparation of RNA for RNA sequencing}

Adult $\mathrm{grna}^{-/-}$and $\mathrm{grna}^{+/+}$control fish (three fish per condition) were subjected to cardiocentesis and kidney dissection as described here. RNA was isolated with RNeasy (Qiagen) following the manufacturer instructions. Total RNA was assessed for quality using an Agilent Tapestation 4200, and samples with an RNA Integrity Number (RIN) greater than 8.0 were used to generate RNA sequencing libraries using the TruSeq Stranded mRNA Sample Prep (Illumina, San Diego, CA). Samples were processed following manufacturer's instructions, starting with 50 ng of RNA and modifying RNA shear time to five minutes. Resulting libraries were multiplexed and 
sequenced with 75 basepair (bp) single reads (SR75) to a depth of approximately 20 million reads per sample on an Illumina HiSeq 4000. Samples were demuxltiplexed using bcl2fastq v2.20 Conversion Software (Illumina, San Diego, CA).

\section{Processing of RNA-seq dataset}

RNA-seq data was mapped to Reference Consortium Zebrafish Build 10 (UCSC Genome GRC10/danRer10; Sept 2014) using Olego (Wu et al, 2013) and normalized using standard analysis pipelined such as cufflinks (Trapnell et al, 2009; Trapnell et al, 2012; Trapnell et al, 2010). feautreCounts (Liao et al, 2014) from subread package is used to compute the raw read counts for each gene. TPM (Transcripts Per Millions) (Li \& Dewey, 2011; Pachter, 2011) values were computed from the raw read counts using a custom perl script and $\log 2(\mathrm{TPM}+1)$ is used to compute the final log-reduced expression values. DESeq2 1.26.0 (Love et al, 2014) R package is used to compute differentially expressed genes at $1 \%$ false discovery rate. Volcano plot and heatmap were created using python matplotlib package (version 2.1.1).

\section{Statistical analysis}

Data were analyzed by unpaired T-test in GraphPad Prism 8. In all figures, solid red bars denote the mean, and error bars represent S.E.M. * $\mathrm{p}<0.05$, ** $\mathrm{p}<0.01$, *** $\mathrm{p}<0.001$, **** $\mathrm{p}<$ 0.0001, n.s. not significant, n.d. not detected.

\section{Acknowledgments}

This work was supported by the NIH-NIDDK K01 (7K01DK115661) and R03 (R03DK125661) awards, Iowa State University start-up funds, postdoctoral fellowship from Fundacion Seneca, Agencia de Ciencia y Tecnologıa de la Region de Murcia, and American Heart Association (16POST30690005) to R.E-P. We thank Roy J. Carver Charitable Trust for the zebrafish 
research facility in the Advanced Teaching and Research Building at ISU. We thank Karen Ong

for technical assistance, and Jesus Olvera and Cody Fine of the UCSD Human Embryonic Stem

Cell Core Facility for technical assistance of flow cytometry experiments. This work was made

possible in part by the CIRM Major Facilities grant (FA1-00607) to the Sanford Consortium for

Regenerative Medicine. We are grateful to Kristen Jepsen, Eugenia Ricciardelli, and Stephanie

Hadimulia of the Institute of Genomics Medicine (IGM) at UCSD for technical assistance of

RNA-sequencing experiments. This publication includes data generated at the UC San Diego

IGM Genomics Center utilizing an Illumina NovaSeq 6000 that was purchased with funding

from a National Institutes of Health SIG grant (\#S10 OD026929).

\section{Authorship contributions}

R.E.-P, C.C, O.F, D.T. designed experiments; R.E.-P, C.C., O.F., E.S, X.C, L.L, A.M, B.S, M.M., performed research; R.E.-P, C.C, X.C, O.F., E.S, L.L, A.M, B.S, D.S, M.M., D.T. analyzed data; and R.E.-P., C.C., and D.T. wrote the paper with minor contributions from remaining authors.

\section{Conflict of Interest Disclosures}

The authors declare no conflicts of interest.

\section{References}

Alquezar C, de la Encarnacion A, Moreno F, Lopez de Munain A, Martin-Requero A (2016) Progranulin deficiency induces overactivation of WNT5A expression via TNF-alpha/NF-kappaB pathway in peripheral cells from frontotemporal dementia-linked granulin mutation carriers. J Psychiatry Neurosci 41: 225-239

Arechavaleta-Velasco F, Perez-Juarez CE, Gerton GL, Diaz-Cueto L (2017) Progranulin and its biological effects in cancer. Med Oncol 34: 194

Bachiller S, Jimenez-Ferrer I, Paulus A, Yang Y, Swanberg M, Deierborg T, Boza-Serrano A (2018) Microglia in Neurological Diseases: A Road Map to Brain-Disease Dependent-Inflammatory Response. Frontiers in cellular neuroscience 12: 488 
Bailey TL, Boden M, Buske FA, Frith M, Grant CE, Clementi L, Ren J, Li WW, Noble WS (2009) MEME SUITE: tools for motif discovery and searching. Nucleic Acids Res 37: W202-208

Bateman A, Belcourt D, Bennett H, Lazure C, Solomon S (1990) Granulins, a novel class of peptide from leukocytes. Biochem Biophys Res Commun 173: 1161-1168

Bateman A, Cheung ST, Bennett HPJ (2018) A Brief Overview of Progranulin in Health and Disease. Methods in molecular biology 1806: 3-15

Bertrand JY, Chi NC, Santoso B, Teng S, Stainier DY, Traver D (2010) Haematopoietic stem cells derive directly from aortic endothelium during development. Nature 464: 108-111

Bhandari V, Giaid A, Bateman A (1993) The complementary deoxyribonucleic acid sequence, tissue distribution, and cellular localization of the rat granulin precursor. Endocrinology 133: 2682-2689

Bhandari V, Palfree RG, Bateman A (1992) Isolation and sequence of the granulin precursor cDNA from human bone marrow reveals tandem cysteine-rich granulin domains. Proc Natl Acad Sci U S A 89: 17151719

Boatman S, Barrett F, Satishchandran S, Jing L, Shestopalov I, Zon LI (2013) Assaying hematopoiesis using zebrafish. Blood Cells Mol Dis 51: 271-276

Brend T, Holley SA (2009) Zebrafish whole mount high-resolution double fluorescent in situ hybridization. Journal of visualized experiments : JoVE

Bright F, Werry EL, Dobson-Stone C, Piguet O, Ittner LM, Halliday GM, Hodges JR, Kiernan MC, Loy CT, Kassiou M et al (2019) Neuroinflammation in frontotemporal dementia. Nat Rev Neurol 15: 540-555

Burda P, Laslo P, Stopka T (2010) The role of PU.1 and GATA-1 transcription factors during normal and leukemogenic hematopoiesis. Leukemia 24: 1249-1257

Cadieux B, Chitramuthu BP, Baranowski D, Bennett HP (2005) The zebrafish progranulin gene family and antisense transcripts. BMC genomics 6: 156

Chantry D, DeMaggio AJ, Brammer H, Raport CJ, Wood CL, Schweickart VL, Epp A, Smith A, Stine JT, Walton K et al (1998) Profile of human macrophage transcripts: insights into macrophage biology and identification of novel chemokines. Journal of leukocyte biology 64: 49-54

Chen YH, Wang YH, Chang MY, Lin CY, Weng CW, Westerfield M, Tsai HJ (2007) Multiple upstream modules regulate zebrafish myf5 expression. BMC developmental biology 7: 1

Chitramuthu BP, Bennett HPJ, Bateman A (2017) Progranulin: a new avenue towards the understanding and treatment of neurodegenerative disease. Brain : a journal of neurology 140: 3081-3104

Daniel R, He Z, Carmichael KP, Halper J, Bateman A (2000) Cellular localization of gene expression for progranulin. The journal of histochemistry and cytochemistry : official journal of the Histochemistry Society 48: 999-1009

de Jong JL, Zon LI (2005) Use of the zebrafish system to study primitive and definitive hematopoiesis. Annu Rev Genet 39: 481-501

de la Encarnacion A, Alquezar C, Martin-Requero A (2016) Increased Wnt Signaling and Reduced Viability in a Neuronal Model of Progranulin-Deficient Frontotemporal Lobar Degeneration. Molecular neurobiology 53: 7107-7118

Ellett F, Pase L, Hayman JW, Andrianopoulos A, Lieschke GJ (2011) mpeg1 promoter transgenes direct macrophage-lineage expression in zebrafish. Blood 117: e49-56

Espin-Palazon R, Stachura DL, Campbell CA, Garcia-Moreno D, Del Cid N, Kim AD, Candel S, Meseguer J, Mulero V, Traver D (2014) Proinflammatory signaling regulates hematopoietic stem cell emergence. Cell 159: 1070-1085

Espin R, Roca FJ, Candel S, Sepulcre MP, Gonzalez-Rosa JM, Alcaraz-Perez F, Meseguer J, Cayuela ML, Mercader N, Mulero V (2013) TNF receptors regulate vascular homeostasis in zebrafish through a caspase-8, caspase-2 and P53 apoptotic program that bypasses caspase-3. Dis Model Mech 6: 383-396

Farag AGA, Shoaib MA, Samaka RM, Abdou AG, Mandour MM, Ibrahim RAL (2019) Progranulin and beta-catenin in psoriasis: An immunohistochemical study. J Cosmet Dermatol 18: 2019-2026

Ganis JJ, Hsia N, Trompouki E, de Jong JL, DiBiase A, Lambert JS, Jia Z, Sabo PJ, Weaver M, Sandstrom R et al (2012) Zebrafish globin switching occurs in two developmental stages and is controlled by the LCR. Dev Biol 366: 185-194 
Hall C, Flores MV, Storm T, Crosier K, Crosier P (2007) The zebrafish lysozyme C promoter drives myeloid-specific expression in transgenic fish. BMC developmental biology 7: 42

Hashimoto S, Suzuki T, Dong HY, Nagai S, Yamazaki N, Matsushima K (1999) Serial analysis of gene expression in human monocyte-derived dendritic cells. Blood 94: 845-852

He Q, Zhang C, Wang L, Zhang P, Ma D, Lv J, Liu F (2015) Inflammatory signaling regulates hematopoietic stem and progenitor cell emergence in vertebrates. Blood 125: 1098-1106

He Z, Ismail A, Kriazhev L, Sadvakassova G, Bateman A (2002) Progranulin (PC-cell-derived growth factor/acrogranin) regulates invasion and cell survival. Cancer Res 62: 5590-5596

He Z, Ong CH, Halper J, Bateman A (2003) Progranulin is a mediator of the wound response. Nat Med 9: 225-229

Heneka MT (2019) Microglia take centre stage in neurodegenerative disease. Nat Rev Immunol 19: 79-80 Hwang HJ, Jung TW, Hong HC, Choi HY, Seo JA, Kim SG, Kim NH, Choi KM, Choi DS, Baik SH et al (2013) Progranulin protects vascular endothelium against atherosclerotic inflammatory reaction via Akt/eNOS and nuclear factor-kappaB pathways. PLoS One 8: e76679

Iwasaki H, Akashi K (2007) Myeloid lineage commitment from the hematopoietic stem cell. Immunity 26: 726-740

Jian J, Konopka J, Liu C (2013) Insights into the role of progranulin in immunity, infection, and inflammation. Journal of leukocyte biology 93: 199-208

Kurotaki D, Nakabayashi J, Nishiyama A, Sasaki H, Kawase W, Kaneko N, Ochiai K, Igarashi K, Ozato K, Suzuki Y et al (2018) Transcription Factor IRF8 Governs Enhancer Landscape Dynamics in Mononuclear Phagocyte Progenitors. Cell Rep 22: 2628-2641

Kwack KH, Lee HW (2017) Progranulin Inhibits Human T Lymphocyte Proliferation by Inducing the Formation of Regulatory T Lymphocytes. Mediators of inflammation 2017: 7682083

Lachmann A, Xu H, Krishnan J, Berger SI, Mazloom AR, Ma'ayan A (2010) ChEA: transcription factor regulation inferred from integrating genome-wide ChIP-X experiments. Bioinformatics 26: 2438-2444

Li B, Dewey CN (2011) RSEM: accurate transcript quantification from RNA-Seq data with or without a reference genome. BMC Bioinformatics 12: 323

Li L, Jin H, Xu J, Shi Y, Wen Z (2011) Irf8 regulates macrophage versus neutrophil fate during zebrafish primitive myelopoiesis. Blood 117: 1359-1369

Li L, Yan B, Shi YQ, Zhang WQ, Wen ZL (2012) Live imaging reveals differing roles of macrophages and neutrophils during zebrafish tail fin regeneration. J Biol Chem 287: 25353-25360

Li Y, Esain V, Teng L, Xu J, Kwan W, Frost IM, Yzaguirre AD, Cai X, Cortes M, Maijenburg MW et al (2014) Inflammatory signaling regulates embryonic hematopoietic stem and progenitor cell production. Genes \& development 28: 2597-2612

Li YH, Chen MH, Gong HY, Hu SY, Li YW, Lin GH, Lin CC, Liu W, Wu JL (2010) Progranulin Amediated MET signaling is essential for liver morphogenesis in zebrafish. J Biol Chem 285: 41001-41009 Liao Y, Smyth GK, Shi W (2014) featureCounts: an efficient general purpose program for assigning sequence reads to genomic features. Bioinformatics 30: 923-930

Liu CJ (2011) Progranulin: a promising therapeutic target for rheumatoid arthritis. FEBS Lett 585: 36753680

Love MI, Huber W, Anders S (2014) Moderated estimation of fold change and dispersion for RNA-seq data with DESeq2. Genome biology 15: 550

Lu R, Serrero G (2001) Mediation of estrogen mitogenic effect in human breast cancer MCF-7 cells by PC-cell-derived growth factor (PCDGF/granulin precursor). Proc Natl Acad Sci U S A 98: 142-147

Lui H, Zhang J, Makinson SR, Cahill MK, Kelley KW, Huang HY, Shang Y, Oldham MC, Martens LH, Gao F et al (2016) Progranulin Deficiency Promotes Circuit-Specific Synaptic Pruning by Microglia via Complement Activation. Cell 165: 921-935

Meers MP, Bryson TD, Henikoff JG, Henikoff S (2019) Improved CUT\&RUN chromatin profiling tools. eLife 8

Minutti CM, Knipper JA, Allen JE, Zaiss DM (2017) Tissue-specific contribution of macrophages to wound healing. Semin Cell Dev Biol 61: 3-11 
Miskolci V, Squirrell J, Rindy J, Vincent W, Sauer JD, Gibson A, Eliceiri KW, Huttenlocher A (2019) Distinct inflammatory and wound healing responses to complex caudal fin injuries of larval zebrafish. eLife 8

Mitroulis I, Kalafati L, Hajishengallis G, Chavakis T (2018) Myelopoiesis in the Context of Innate Immunity. Journal of innate immunity 10: 365-372

Monteiro R, Pouget C, Patient R (2011) The gata1/pu.1 lineage fate paradigm varies between blood populations and is modulated by tif1gamma. EMBO J 30: 1093-1103

Morales RA, Allende ML (2019) Peripheral Macrophages Promote Tissue Regeneration in Zebrafish by Fine-Tuning the Inflammatory Response. Frontiers in immunology 10: 253

Nguyen-Chi M, Laplace-Builhe B, Travnickova J, Luz-Crawford P, Tejedor G, Lutfalla G, Kissa K, Jorgensen C, Djouad F (2017) TNF signaling and macrophages govern fin regeneration in zebrafish larvae. Cell death \& disease 8: e2979

Olsson A, Venkatasubramanian M, Chaudhri VK, Aronow BJ, Salomonis N, Singh H, Grimes HL (2016) Single-cell analysis of mixed-lineage states leading to a binary cell fate choice. Nature 537: 698-702

Ong CH, He Z, Kriazhev L, Shan X, Palfree RG, Bateman A (2006) Regulation of progranulin expression in myeloid cells. American journal of physiology Regulatory, integrative and comparative physiology 291: R1602-1612

Pachter L (2011) Models for transcript quantification from RNA-Seq. arXiv 1104.3889

Paik EJ, Zon LI (2010) Hematopoietic development in the zebrafish. Int J Dev Biol 54: 1127-1137

Pathria P, Louis TL, Varner JA (2019) Targeting Tumor-Associated Macrophages in Cancer. Trends in immunology 40: 310-327

Petkau TL, Leavitt BR (2014) Progranulin in neurodegenerative disease. Trends Neurosci 37: 388-398

Ray-Gallet D, Mao C, Tavitian A, Moreau-Gachelin F (1995) DNA binding specificities of Spi-1/PU.1 and Spi-B transcription factors and identification of a Spi-1/Spi-B binding site in the c-fes/c-fps promoter. Oncogene 11: 303-313

Renshaw SA, Loynes CA, Trushell DM, Elworthy S, Ingham PW, Whyte MK (2006) A transgenic zebrafish model of neutrophilic inflammation. Blood 108: 3976-3978

Rhodes J, Hagen A, Hsu K, Deng M, Liu TX, Look AT, Kanki JP (2005) Interplay of pu.1 and gata1 determines myelo-erythroid progenitor cell fate in zebrafish. Dev Cell 8: 97-108

Rouillard AD, Gundersen GW, Fernandez NF, Wang Z, Monteiro CD, McDermott MG, Ma'ayan A (2016) The harmonizome: a collection of processed datasets gathered to serve and mine knowledge about genes and proteins. Database (Oxford) 2016

Seita J, Sahoo D, Rossi DJ, Bhattacharya D, Serwold T, Inlay MA, Ehrlich LI, Fathman JW, Dill DL, Weissman IL (2012) Gene Expression Commons: an open platform for absolute gene expression profiling. PLoS One 7: e40321

Shiau CE, Kaufman Z, Meireles AM, Talbot WS (2015) Differential requirement for irf8 in formation of embryonic and adult macrophages in zebrafish. PLoS One 10: e0117513

Simoes FC, Cahill TJ, Kenyon A, Gavriouchkina D, Vieira JM, Sun X, Pezzolla D, Ravaud C, Masmanian E, Weinberger M et al (2020) Macrophages directly contribute collagen to scar formation during zebrafish heart regeneration and mouse heart repair. Nature communications 11: 600

Skene PJ, Henikoff S (2017) An efficient targeted nuclease strategy for high-resolution mapping of DNA binding sites. eLife 6

Solchenberger B, Russell C, Kremmer E, Haass C, Schmid B (2015) Granulin knock out zebrafish lack frontotemporal lobar degeneration and neuronal ceroid lipofuscinosis pathology. PLoS One 10: e0118956 Stachura DL, Traver D (2011) Cellular dissection of zebrafish hematopoiesis. Methods Cell Biol 101: 75110

Tang Q, Iyer S, Lobbardi R, Moore JC, Chen H, Lareau C, Hebert C, Shaw ML, Neftel C, Suva ML et al (2017) Dissecting hematopoietic and renal cell heterogeneity in adult zebrafish at single-cell resolution using RNA sequencing. J Exp Med 214: 2875-2887 
Tang W, Lu Y, Tian QY, Zhang Y, Guo FJ, Liu GY, Syed NM, Lai Y, Lin EA, Kong L et al (2011) The growth factor progranulin binds to TNF receptors and is therapeutic against inflammatory arthritis in mice. Science 332: 478-484

Theodore LN, Hagedorn EJ, Cortes M, Natsuhara K, Liu SY, Perlin JR, Yang S, Daily ML, Zon LI, North TE (2017) Distinct Roles for Matrix Metalloproteinases 2 and 9 in Embryonic Hematopoietic Stem Cell Emergence, Migration, and Niche Colonization. Stem cell reports 8: 1226-1241

Thisse C, Thisse B, Schilling TF, Postlethwait JH (1993) Structure of the zebrafish snail1 gene and its expression in wild-type, spadetail and no tail mutant embryos. Development 119: 1203-1215

Tian R, Li Y, Yao X (2016) PGRN Suppresses Inflammation and Promotes Autophagy in Keratinocytes Through the Wnt/beta-Catenin Signaling Pathway. Inflammation 39: 1387-1394

Trapnell C, Pachter L, Salzberg SL (2009) TopHat: discovering splice junctions with RNA-Seq. Bioinformatics 25: 1105-1111

Trapnell C, Roberts A, Goff L, Pertea G, Kim D, Kelley DR, Pimentel H, Salzberg SL, Rinn JL, Pachter L (2012) Differential gene and transcript expression analysis of RNA-seq experiments with TopHat and Cufflinks. Nature protocols 7: 562-578

Trapnell C, Williams BA, Pertea G, Mortazavi A, Kwan G, van Baren MJ, Salzberg SL, Wold BJ, Pachter L (2010) Transcript assembly and quantification by RNA-Seq reveals unannotated transcripts and isoform switching during cell differentiation. Nat Biotechnol 28: 511-515

Traver D, Paw BH, Poss KD, Penberthy WT, Lin S, Zon LI (2003) Transplantation and in vivo imaging of multilineage engraftment in zebrafish bloodless mutants. Nat Immunol 4: 1238-1246

Traver D, Winzeler A, Stern HM, Mayhall EA, Langenau DM, Kutok JL, Look AT, Zon LI (2004) Effects of lethal irradiation in zebrafish and rescue by hematopoietic cell transplantation. Blood 104: 1298-1305

Travnickova J, Tran Chau V, Julien E, Mateos-Langerak J, Gonzalez C, Lelievre E, Lutfalla G, Tavian M, Kissa K (2015) Primitive macrophages control HSPC mobilization and definitive haematopoiesis. Nature communications 6: 6227

Wagner DE, Weinreb C, Collins ZM, Briggs JA, Megason SG, Klein AM (2018) Single-cell mapping of gene expression landscapes and lineage in the zebrafish embryo. Science 360: 981-987

Ward ME, Chen R, Huang HY, Ludwig C, Telpoukhovskaia M, Taubes A, Boudin H, Minami SS, Reichert M, Albrecht P et al (2017) Individuals with progranulin haploinsufficiency exhibit features of neuronal ceroid lipofuscinosis. Sci Transl Med 9

Wei F, Zhang Y, Zhao W, Yu X, Liu CJ (2014) Progranulin facilitates conversion and function of regulatory T cells under inflammatory conditions. PLoS One 9: e112110

Wei JL, Fu W, Ding YJ, Hettinghouse A, Lendhey M, Schwarzkopf R, Kennedy OD, Liu CJ (2017) Progranulin derivative Atsttrin protects against early osteoarthritis in mouse and rat models. Arthritis Res Ther 19: 280

Westerfield M (2000) The Zebrafish Book. A Guide for the Laboratory Use of Zebrafish Danio* (Brachydanio) rerio. . University of Oregon Press., Eugene, OR.

Wolff L, Humeniuk R (2013) Concise review: erythroid versus myeloid lineage commitment: regulating the master regulators. Stem cells 31: 1237-1244

Wu J, Anczukow O, Krainer AR, Zhang MQ, Zhang C (2013) OLego: fast and sensitive mapping of spliced mRNA-Seq reads using small seeds. Nucleic Acids Res 41: 5149-5163

Wynn TA, Chawla A, Pollard JW (2013) Macrophage biology in development, homeostasis and disease. Nature 496: 445-455

Yin F, Banerjee R, Thomas B, Zhou P, Qian L, Jia T, Ma X, Ma Y, Iadecola C, Beal MF et al (2010) Exaggerated inflammation, impaired host defense, and neuropathology in progranulin-deficient mice. $J$ Exp Med 207: 117-128

Zambusi A, Pelin Burhan O, Di Giaimo R, Schmid B, Ninkovic J (2020) Granulins Regulate Aging Kinetics in the Adult Zebrafish Telencephalon. Cells 9

Zanocco-Marani T, Bateman A, Romano G, Valentinis B, He ZH, Baserga R (1999) Biological activities and signaling pathways of the granulin/epithelin precursor. Cancer Res 59: 5331-5340 
Zerbino DR, Johnson N, Juetteman T, Sheppard D, Wilder SP, Lavidas I, Nuhn M, Perry E, RaffaillacDesfosses Q, Sobral D et al (2016) Ensembl regulation resources. Database (Oxford) 2016

Zhang P, Zhang X, Iwama A, Yu C, Smith KA, Mueller BU, Narravula S, Torbett BE, Orkin SH, Tenen DG (2000) PU.1 inhibits GATA-1 function and erythroid differentiation by blocking GATA-1 DNA binding. Blood 96: 2641-2648

Zhu J, Nathan C, Jin W, Sim D, Ashcroft GS, Wahl SM, Lacomis L, Erdjument-Bromage H, Tempst P, Wright CD et al (2002) Conversion of proepithelin to epithelins: roles of SLPI and elastase in host defense and wound repair. Cell 111: 867-878

\section{Figure Legends}

\section{Figure 1: grna expression is restricted to the myeloid cell lineage during embryo}

development. Expression of grna (A) and grnb (B) during zebrafish embryonic and larval development. The mRNA levels were determined by real-time quantitative PCR in 10-30 pooled larvae at the indicated times. The gene expression is normalized against efla, each bar represents the mean \pm S.E. from two independent experiments. (C) Expression of grna (upper panel) and grnb (lower panel) by WISH at 48hpf. Black arrowheads denote grna expression by distinct individual cells. Note that the grnb expression pattern is ubiquitous. Anterior is to the left, dorsal to the top. (D-D') scRNA-seq graph showing grna expression (green dots) using the online tool SPRING by Wagner and colleagues (Wagner et al., 2018). Dots represent single-cells from 4 hpf (center) to $24 \mathrm{hpf}$ (periphery) zebrafish embryos. The cells that expressed grna (green dots) are magnified in D'. Notice that grna expression is restricted to germline cells (green box) and leukocytes (orange box). (E) Muscle $\left(M y f 5: e G F P^{+}\right)$, vasculature (Flk:mcherry ${ }^{+}$; Gatal:Dsred $)$, neutrophils $\left(M p x: e G F P^{+}\right)$, macrophages $\left(M p e g 1: e G F P^{+}\right)$, and erythrocytes (Globin:eGFP $\left.{ }^{+}\right)$ cells from dissected embryos were purified by FACS, and qPCR for grna performed. Levels of grna transcripts along $\mathrm{x}$-axis are shown relative to the housekeeping gene efla. Bars represent means \pm S.E.M. of two to three independent samples. ND, not detected; hpf, hours postfertilization. 


\section{Figure 2: Absence of grna leads to decreased myeloid differentiation during embryo}

development. (A) Representative fluorescence images of the tails of 48 hpf Mpeg1:eGFP; Lyz:dsred double transgenic embryos injected with Grna mismatch control, Grna or Grnb MOs.

(B, B'-F, F') WISH for the myeloid progenitor (pu.1), macrophage (mfap4) and neutrophilic (mpx) markers in grna $^{-/-}$and $g r n a^{+/+}$control embryos at $48 \mathrm{hpf}$ (B, B''-E, E'') or 5 dpf (F-F'). Black arrowheads depict cells expressing the indicated transcripts. (B', C', D', E', F',) Enumeration of $\mathrm{puI}^{+}, \mathrm{mfap}^{+}, \mathrm{mpx}^{+}$and $a p o e b^{+}$expressing cells shown in (B,B'- F, F'). Each dot represents the number of positive cells in the photographed area in each embryo. Bars represent mean \pm S.E.M. Ns, not significant. $* p<0.05$, and $* * * p<0.001$.

Figure 3: Granulin expression is up-regulated in vertebrate myeloid cells and is essential for myeloid cell differentiation during adult hematopoiesis. (A) tSNE analysis showing grna expression levels (red is high, orange and yellow are medium expression; blue is absent) of single cells sequenced from wildtype zebrafish kidney marrow using the online visualizer "Single Cell inDrops RNA-Seq Visualization of Adult Zebrafish Whole Kidney Marrow" (https://molpath.shinyapps.io/zebrafishblood/\#pltly) (Tang et al., 2017). The main tSNE clusters identified expressing grna are denoted by open circles. Yellow open circles represent clusters defined as "macrophages". Open green circles are grna expressing clusters whose cells were identified as "myeloid cells". (B) Mouse hematopoietic model showing the dynamic expression of Grn derived from microarray data (Affymetrix Mouse Genome 4302.0 Array). Notice that lymphocyte differentiation beyond CLP is not shown here. (C) Representative flow cytometric light scatter profile showing the different hematopoietic populations present in $\mathrm{grna}^{+/+}$(left) and grna $^{-/}$(right) kidney marrow. (D) Representative pictures from grna $^{+/+}$and $g r n a^{-/}$whole kidney marrows cytospins stained with Wright-Giemsa showing increased early myeloid precursors 
(orange arrowheads) and decreased mature neutrophils (green arrowheads) in the absence of grna (bottom panel) compared to grna $^{+/+}$control siblings (upper panel). (E) Manual quantification of kidney marrow hematopoietic cells in grna $^{-/-}$(green squares, $\mathrm{n}=5$ ) compared to control $\mathrm{grna}^{+/+}$(black dots, $\mathrm{n}=5$ ) from two independent experiments. Horizontal lines and error bars indicate means \pm SEM $(* * * p<0.001, * * * * p<0.0001)$. HSC, Hematopoietic Stem Cell; MPP, Hematopoietic multipotential progenitors; GMLP, granulocyte-monocyte-lymphoid progenitor; CLP, common lymphoid progenitor; pMEP, pre of megakaryocyte-erythroid progenitor; MEP, megakaryocyte-erythroid progenitor; MkP, Megakaryocyte progenitor; PCFU-e, Colony Forming Unit-Erythroid; Plt, platelets; Ery, Erythrocytes; sCMP, strict common myeloid progenitor; pGMP, pre-granulocyte/macrophage; GMP, granulocyte/macrophage progenitors; Gra Gr+, granulocytes; Mono, monocytes.

Figure 4: Grna inhibits erythroid differentiation. (A) RNA-seq analysis from grna $^{-/}$and grna $^{+/+}$adult zebrafish kidney marrows reveals 154 down-regulated and 116 up-regulated genes in grna $^{-/-}$versus grna $^{+/+}$control. (B) Volcano plot obtained from DESeq2 analysis of grna ${ }^{-/-}$and $g_{r n a}^{+/+}$kidney marrows. (C) Heat map of the enriched and depleted transcripts in kidney marrows from $\mathrm{grna}^{-/-}$versus $\mathrm{grna}^{+/+}$adult fish. Color-coding is based on rlog-transformed read count values. (D) Fluorescence images of gatala:Dsred embryos injected with Grna morpholino (Grna-MO) (upper panel) and a five-base Grna mismatch control (bottom panel), showing increased gatala:Dsred fluorescence in the absence of Grna. (E) Histogram of three-pooled gatala:Dsred embryos injected with Grna-MO (red) or mismatch Grna-MO control (grey).

Figure 5: Transcriptional factor network that controls granulin expression. (A) Double fluorescence in situ hybridization for grna (red) and pu.l (green) shows colocalization of both transcripts. Nuclei are stained with DAPI (blue). Pictures were taken by confocal microscopy 
from the tail region of $48 \mathrm{hpf}$ zebrafish embryos. Each image is a $1 \mu \mathrm{m} \mathrm{z}$ slice. (B) WISH for the neutrophilic marker mpx (upper panels, arrowheads) or grna (lower panels, arrowheads) after morpholino knockdown of Pu.1 (middle panels) or Irf8 (right panels) compared to Standard morpholino control (left panels) at 48 hpf. Pu.1 or Irf8 knockdown abolished grna expression. Asterisks denote natural pigmentation occurring in the tail of zebrafish embryos. (C) Schematic representation of the grna gene and its 5' enhancer locus denoting six putative $\mathrm{Pu} .1$ binding sites (BSs). Pu.1 BS1-3 (green squares) were found searching the human pU.1-target site nucleotide matrix represented in (D) using the motif comparison tool Tomtom. Pu.1 BS5-6 (orange squares) were found searching the pU.1-target site nucleotide matrix available in ConSite (http://consite.genereg.net/cgi-bin/consite). BSs positions are denoted with numbers in between brackets. Positive "+" numbering starts with +1 at the A of the grna ATG translation initiation (start) codon. Nucleotides upstream (5') of the grna ATG-translation initiation codon (start) are marked with a "-" (minus). E, exon. White squares with blue line indicate grna exons from the untranslated region. Blue squares represent grna coding exons. Control primers to amplify 71 base pairs of a locus within the grna gene with no predicted $\mathrm{Pu} .1 \mathrm{BSs}$ for CUT\&RUN normalization are indicated with a purple square. (E) Representative CUT\&RUN experiment performed in fresh zebrafish kidney marrows from adult $\mathrm{AB}^{*}$ using a Pu.1 or control IgG antibody. Fold enrichment of Pu.1-associated DNA fragments were identified by qPCR using primers flanking the BSs denoted in (C). To calculate the fold enrichment, qPCR results for each BS were normalized against spike-in DNA as described (Meers et al, 2019) and control primers that amplify a locus of the grna gene lacking predicted Pu.1 BSs. This experiment was performed three independent times with similar enrichments. The primers used are shown in Table S1. 
Figure 6: Macrophages respond abnormally to injury in the absence of Grna. (A) Experimental workflow. Transgenic Mpeg1:eGFP one-cell stage embryos were injected with either GrnaMO or Grna mismatch MO. At 48 hpf, caudal tails were resected immediately after the end of the notochord. Fluorescence imaging of the tail (CHT, were the majority of neutrophils reside at this developmental time, and wound area) was taken every two hours from 1 hpw to $32 \mathrm{hpw}$, and the number of neutrophils quantified manually. (B) Neutrophil numbers in the CHT from individual Mpegl:eGFP transgenic animals at 1, 3, 5, 7 and $9 \mathrm{hpw}$ following depletion of Grna compared to Grna mismatch control morphants. (C) Percentage of Mpegl:eGFP ${ }^{+}$macrophages recruited to the wound region normalized to the total macrophage number in the tail (CHT and wound) in GrnaMO (red line, n=3) and Grna mismatch MO (blue line, $n=3$ ) injected embryos at indicated time points. Circle and square dots indicate means, and error bars SEM (ns, not significant; *p<0.05, **p<0.001). (D) Representative fluorescence images of tail fins from Mpeg1:eGFP Grna- or Grna mismatch control morphant siblings at the indicated times.

Figure 7: Grna mutants fail to regenerate the tail fin after resection. (A) Experimental workflow. $\mathrm{grna}^{+/+}$or $\mathrm{grna}^{-/-} 48 \mathrm{hpf}$ embryos were subjected to caudal tail resection immediately after the end of the notochord. Bright field imaging of the wound was taken every 24 hours for three days (72 hpw, equivalent to $120 \mathrm{hpf}$ ). (B) Representative images of fin tails from $\mathrm{grna}^{+/+}$ (top panel) or grna $^{-/}$(bottom panel) larvae at the indicated times. Black lines indicate where the fin tails were resected. (C) Quantification of the regenerated tail fin area of $\operatorname{grna}^{+/+}(\mathrm{n}=5)$ and grna $^{-/}(\mathrm{n}=5)$ larvae from (B). $* * \mathrm{p}<0.001, * * * \mathrm{p}<0.0001$. (D) Magnification of regenerated tail fins from $\mathrm{grna}^{+/+}$and $\mathrm{grna}^{-/-}$larvae (black rectangles in B). To facilitate collagen fivers visualization, raw pictures were contrasted automatically using the "contrast enhancement" tool 
of CorelDraw version X6. (E) Schematic representation of signaling occurring during myeloid cell differentiation. Briefly, Pu.1 and Irf8 positively regulate granulin expression, which in turn controls the expression of rgs 2 and cebpa for the differentiation of myeloid progenitors into neutrophils expressing mpx and lyz or macrophages (mpegl and mfap4). Granulin blocks gatal expression, inhibiting erythroid development and the expression of the erythroid related genes alas2, epb41b and hbae4. Granulin expression levels are indicated in green.

\section{Supplementary Figure 1: grna and grnb transcripts are ubiquitously expressed during} early zebrafish embryonic development. WISH of 2, 6 and $10 \mathrm{hpf} \mathrm{AB}^{*}$ zebrafish embryos hybridized with grna antisense and control sense probes (left panel), or grnb antisense and control sense probes (right panel). Numbers represent embryos with indicated expression.

\section{Supplementary Figure 2: grna knockdown impairs macrophage development. (A-B)}

Schematic illustration of the grna and grnb knock-out and strategies. (A) Grna-MO1 and GrnaMO2 are 5'UTR-ATG translation blocking morpholinos. (B-C) Efficiency of splice-blocking MO against zebrafish grnb. RT-PCR analysis of WT and Grnb-MO injected embryos induced altered splicing of the grnb transcripts at $24 \mathrm{hpf}$. A $535 \mathrm{bp}$ product containing 37 nucleotides insertion from grnb intron 5 (upper band) was detected in contrast to a 498 bp control (lower band in Grnb-MO injected embryos and WT control). This leads to an aberrant grnb mature mRNA with a shift in reading frame, which results in premature stop codons along the grnb mRNA. The annealing of MOs (green lines) and the inframe premature stop codons (arrowheads) are indicated. (D) Representative dots plots of 36hpf Mpeg1.1:eGFP embryos injected with mismatch, Grna-MO1 and Grna-MO2. (E) Quantification of Mpeg+ macrophages in Mismatch control n=4, Grna-MO1 n=4, and Grna-MO2, n=3 embryos. Each dot represents the percentage of Mpeg+ cells from the total events from 3 embryos. Horizontal lines and error bars 
indicate means \pm SEM $(* * \mathrm{p}<0.01, * * * \mathrm{p}<0.001$, ns: no significant). (F-G) Representative dot plot of intracellular flow cytometry using a specific antibody against the macrophage marker Mfap4 at 48 hpf grna $^{+/+}$(left) or grna ${ }^{-/-}$embryos (right). Quantification shown in (G). (H) WIHC for eGFP (green) and phospho-Histone 3 (Ser10) (red) in the caudal hematopoietic tissue of $30 \mathrm{hpf}$ Mpx:eGFP embryos shows lack of colocalization between both markers.

\section{Supplementary Figure 3: Conserved gene expression levels among zebrafish grna and} mouse Grn in hematopoietic cells. (A) Heat map derived from the online visualizer "Single Cell inDrops RNA-Seq Visualization of Adult Zebrafish Whole Kidney Marrow" (https://molpath.shinyapps.io/zebrafishblood/\#pltly) (Tang et al., 2017) for grna and grnb showing low or inexistent expression of grnb by kidney marrow hematopoietic cells and high expression by multiciliated kidney cells and vascular endothelium. In contrast, grna is highly expressed by macrophages and myeloid cells. (B) Gene expression levels in Transcripts Per Kilobase Million (TPM) for Granulin (Grn, red) and Pu.1 (Spil, blue) from single cell RNA-seq data of mouse hematopoietic cells (Olsson et al., 2016) showing high correlation between Grn and Spil expression. (C) Quantification of the percentage of myeloid cells gated in Figure 3C in grna $^{+/+}(\mathrm{n}=4)$ and grna $^{-/-}(\mathrm{n}=4)$ zebrafish kidney marrows. (D) Quantification of zebrafish body length of $\mathrm{grna}^{-/-}$and $\mathrm{grna}^{+/+}$control siblings. Horizontal lines and error bars indicate means \pm SEM. Ns, not significant; *p $<0.05$. HSCP, Haematopoietic Stem Cell Progenitor; Meg, megakaryocytic; Ery, erythrocytic; Multi, Multi-lineage primed; MDP, monocyte-dendritic cell precursor; Mono, monocytic; Gran, granulocytic; Myelo, myelocyte (myelocytes and metamyelocytes).

Supplementary Figure 4: Grna depletion leads to decreased expression of myeloid specific genes and increased erythroid genes in the kidney marrow. Heat map of the relative gene 
expression derived from the online visualizer "Single Cell inDrops RNA-Seq Visualization of Adult Zebrafish Whole Kidney Marrow” (https://molpath.shinyapps.io/zebrafishblood/\#pltly) (Tang et al., 2017) of genes significantly enriched (A) or depleted (B) from RNA-seq of $g r n a^{-/}$ versus grna $^{+/+}$kidney marrows. Supplementary Figure 5: Conserved regulatory expression of granulin in mammals and zebrafish. (A) Mpeg 1:eGFP transgenic embryos were injected with control (Std), Pu.1, or Irf8 MOs and the tail region was visualized by fluorescence microscopy at $48 \mathrm{hpf}$. (B) Mouse hematopoietic model showing the dynamic expression of Irf8 derived from microarray data (Affymetrix Mouse Genome 4302.0 Array). Notice that lymphocyte differentiation beyond CLP is not shown here. (C) Screenshot of the regulatory feature of ensembl.org showing that PU.1 binds the GRN promoter in human hematopoietic cell lines based on epigenetic marks from experimental data available through ensembl.org Screenshot of mammalian granulin queried by ChEA showing the transcription factors whose peaks were detected at the granulin promoter. TFs were ranked based on p-value (red colors indicate $\mathrm{p}<0,05$; gray colors indicate $\mathrm{p}>0,05$ ). (E) Results from Harmonizome combined with ChEA showing the TFs that were co-expressed with the mammalian granulin. TFs were ranked based on $\mathrm{p}$-value (red colors indicate $\mathrm{p}<0,05)$. (F) Screenshot of mammalian granulin queried by Harmonizome (https://amp.pharm.mssm.edu/archs4/gene/GRN\#correlation) showing the first 10 most similar genes based on co-expression ranked by descendent Pearson correlation. HSC, Hematopoietic Stem Cell; MPP, Hematopoietic multipotential progenitors; GMLP, granulocytemonocyte-lymphoid progenitor; CLP, common lymphoid progenitor; pMEP, pre of megakaryocyte-erythroid progenitor; MEP, megakaryocyte-erythroid progenitor; MkP, Megakaryocyte progenitor; PCFU-e, Colony Forming Unit-Erythroid; Plt, platelets; Ery, 
bioRxiv preprint doi: https://doi.org/10.1101/2020.07.23.217067; this version posted July 23, 2020. The copyright holder for this preprint (which was not certified by peer review) is the author/funder. All rights reserved. No reuse allowed without permission.

Erythrocytes; sCMP, strict common myeloid progenitor; pGMP, pre-granulocyte/macrophage;

GMP, granulocyte/macrophage progenitors; Gra Gr+, granulocytes; Mono, monocytes. 
Figure 1

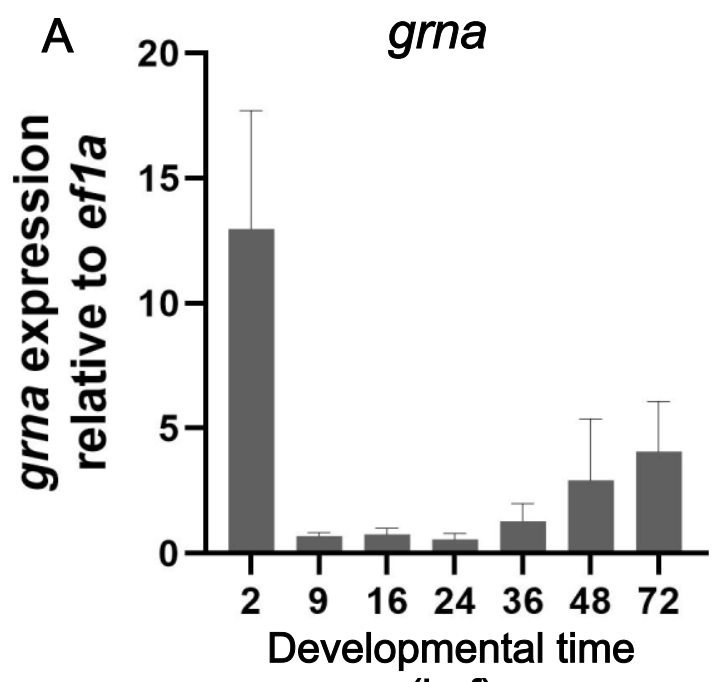

(hpf)

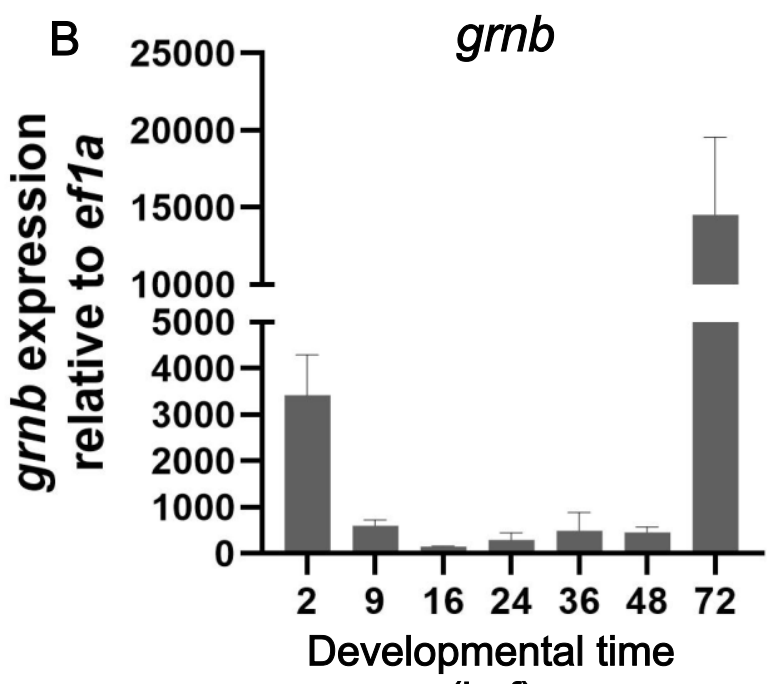

(hpf)

C
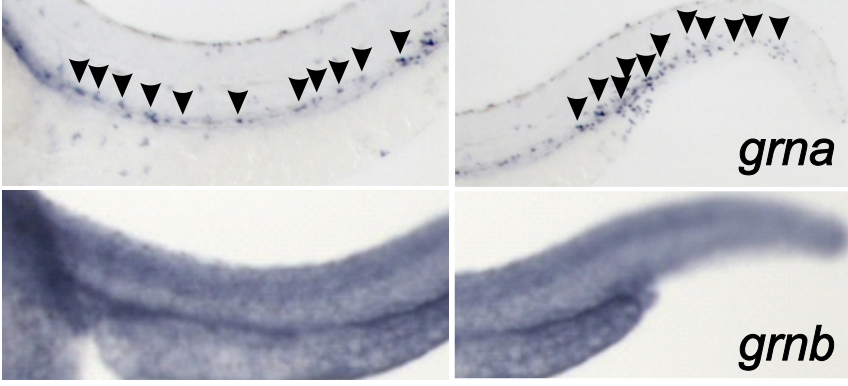

D

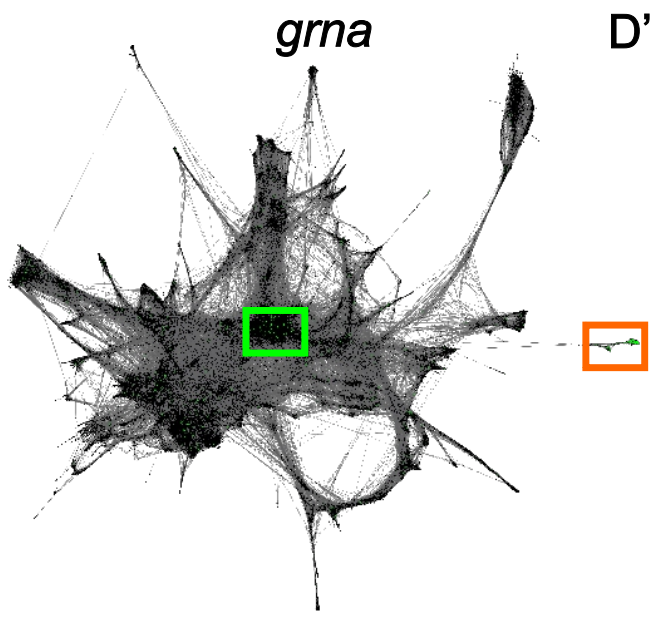

E
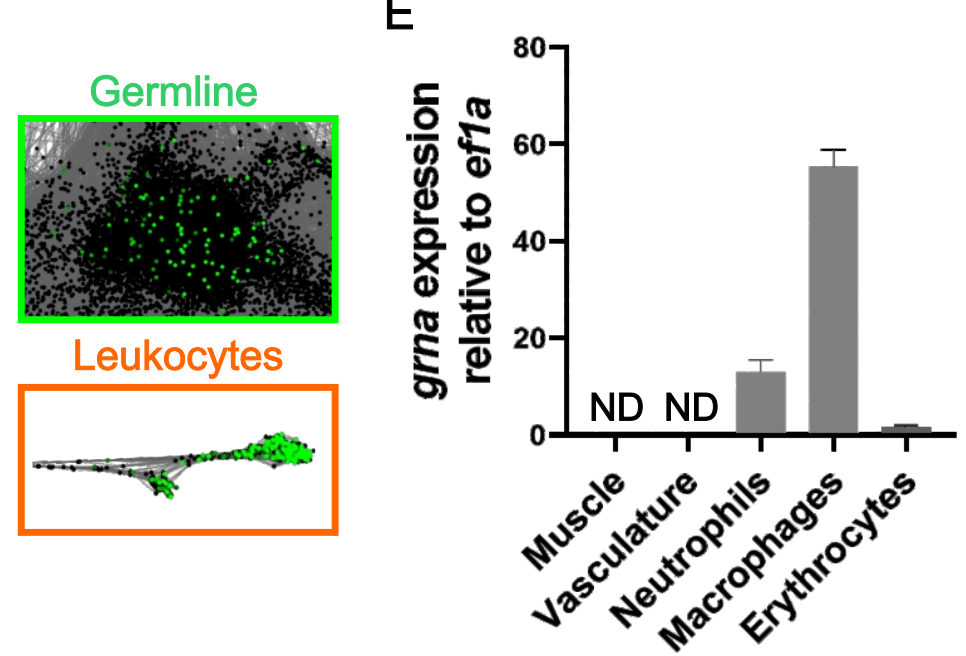


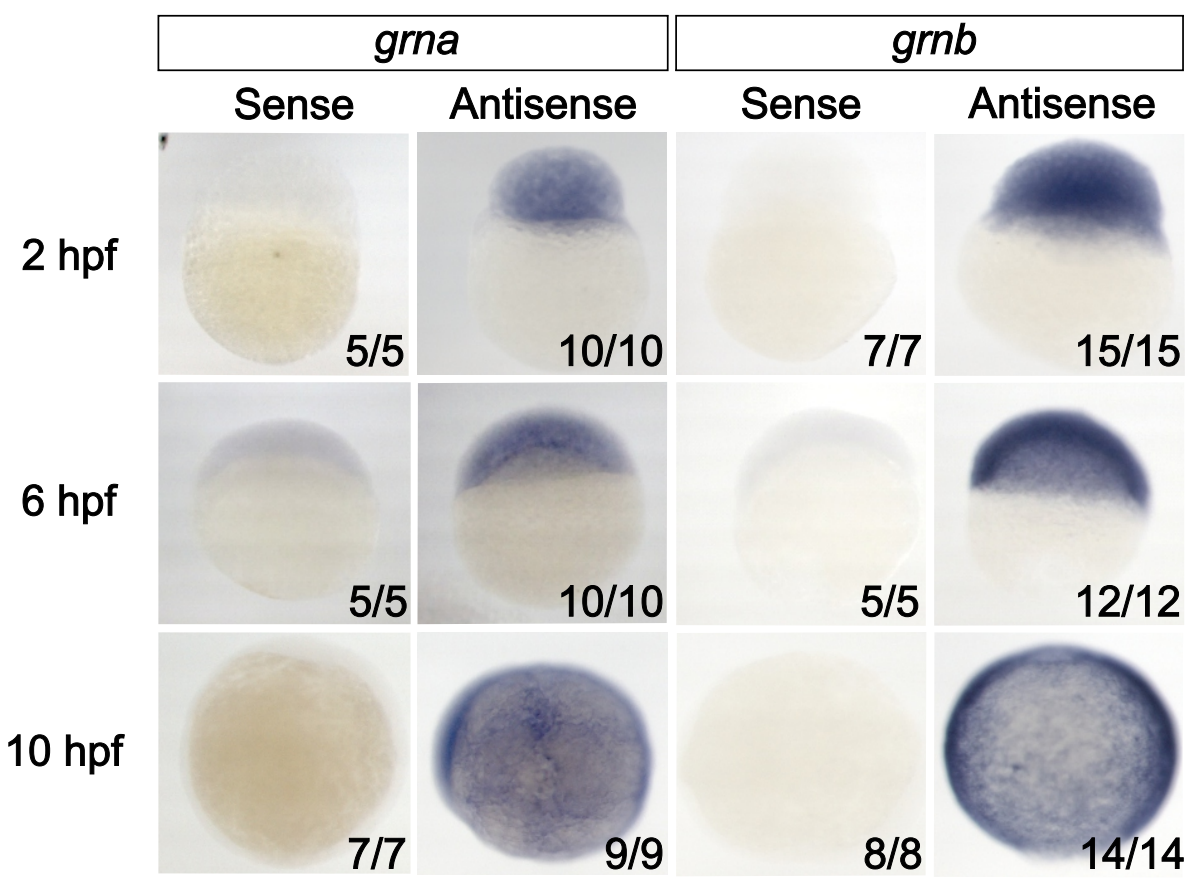



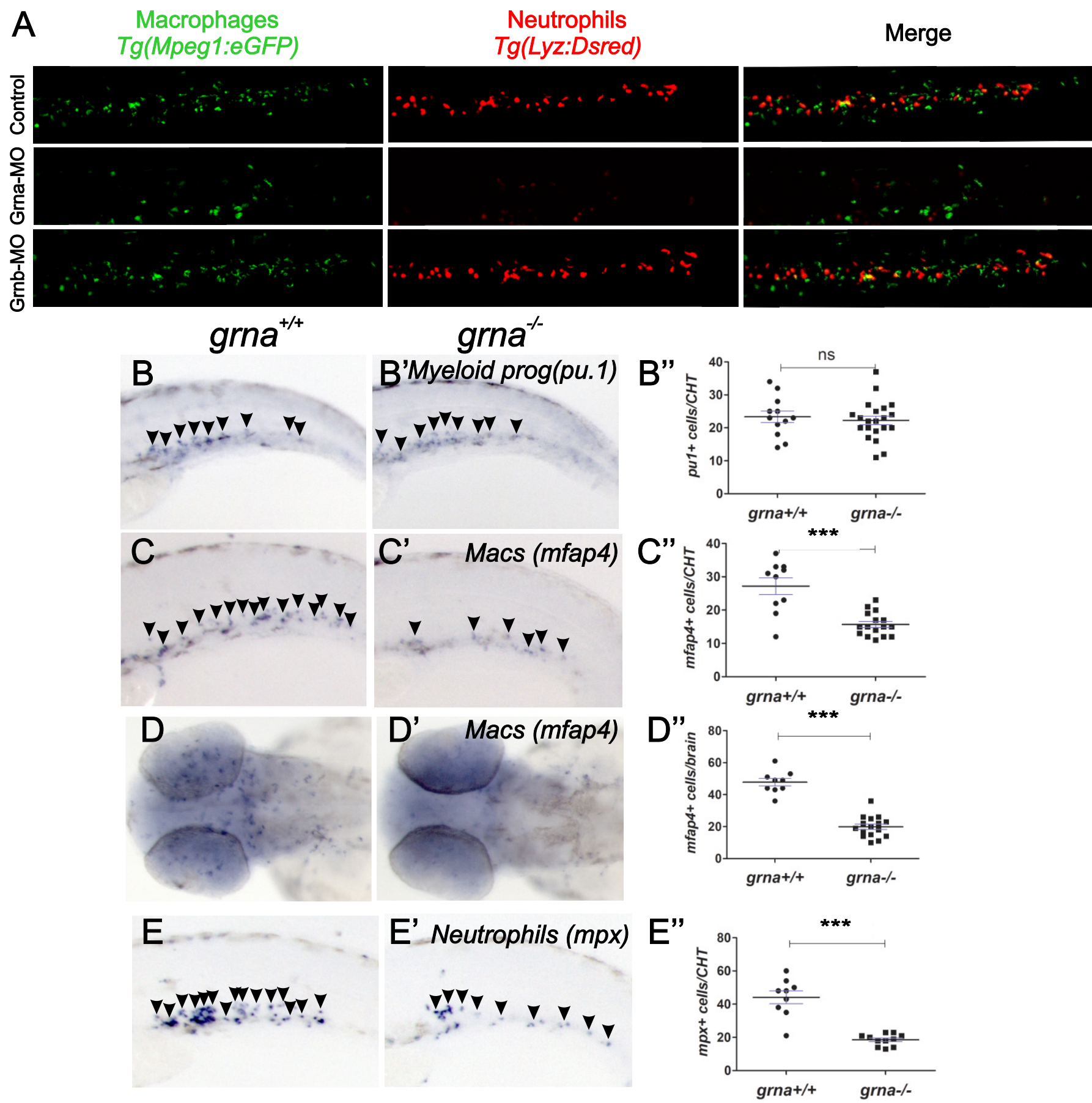
Sup Figure 2

A

grna mRNA

grna coding region

Translation blocking of grna

B
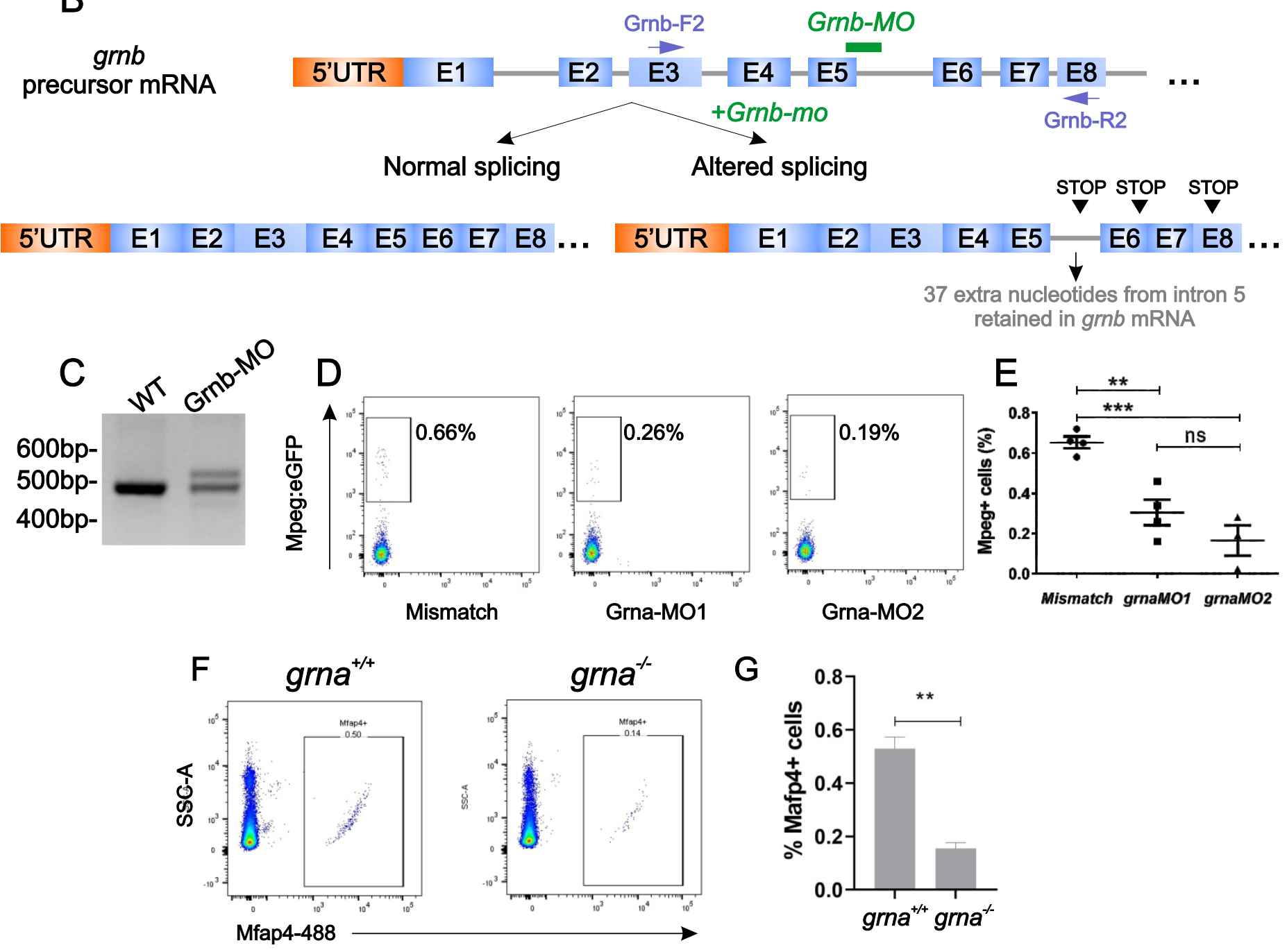

G

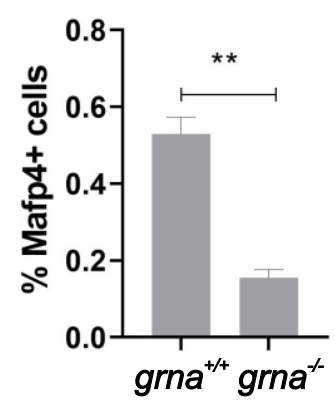

$\mathrm{H}$

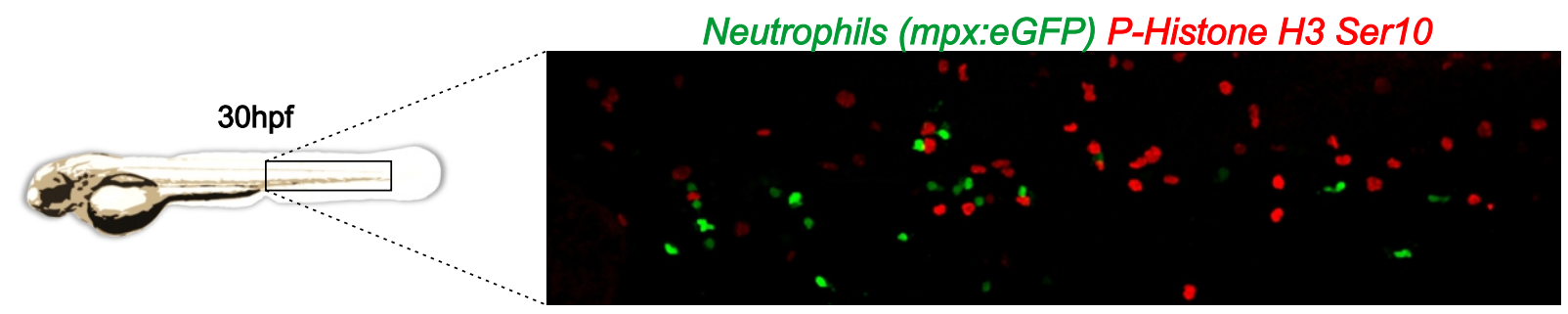


A

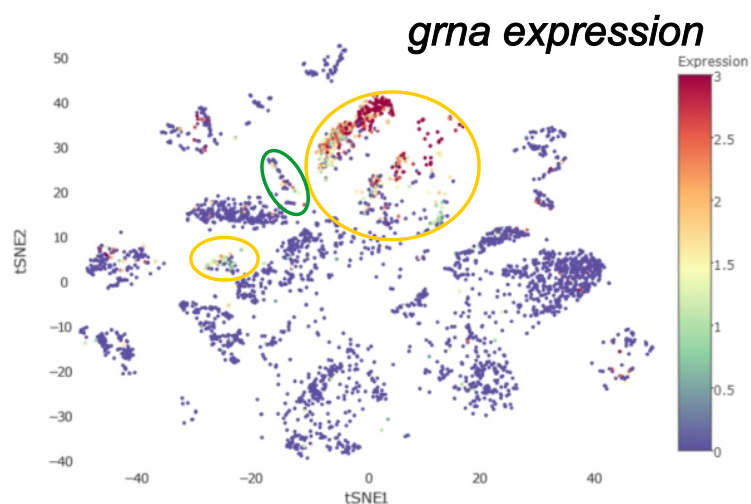

OMyeloid cells $\bigcirc$ Macrophages
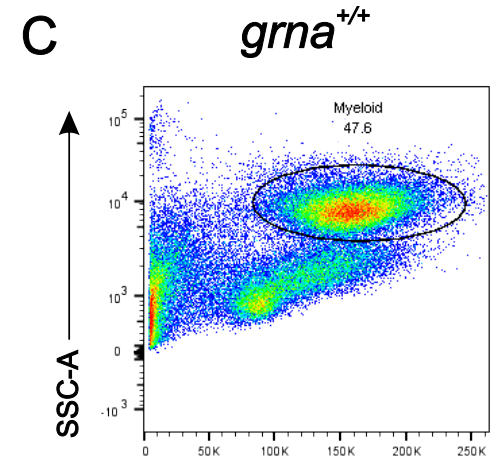

FSC-A
B
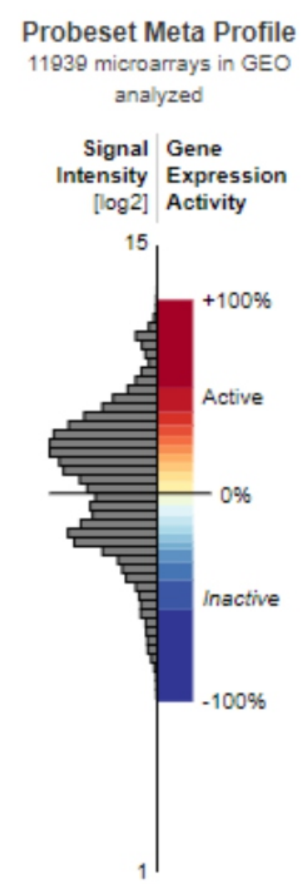

7.33

Dynamic-range [log2]

Figure 3

D

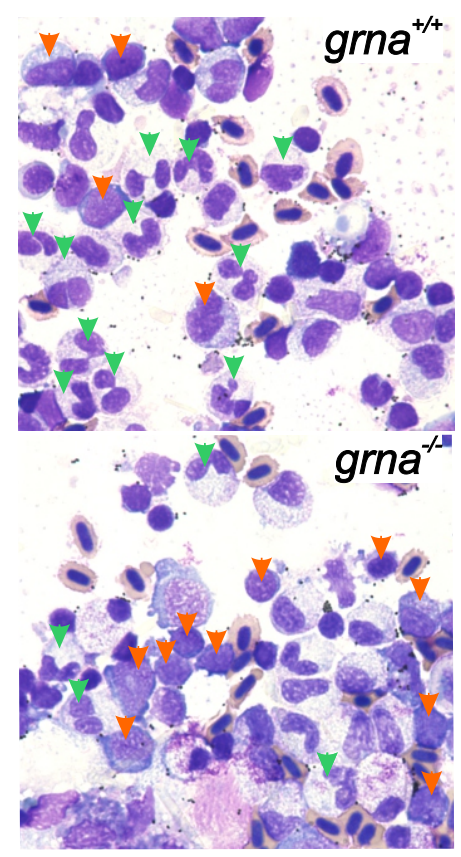

$E$
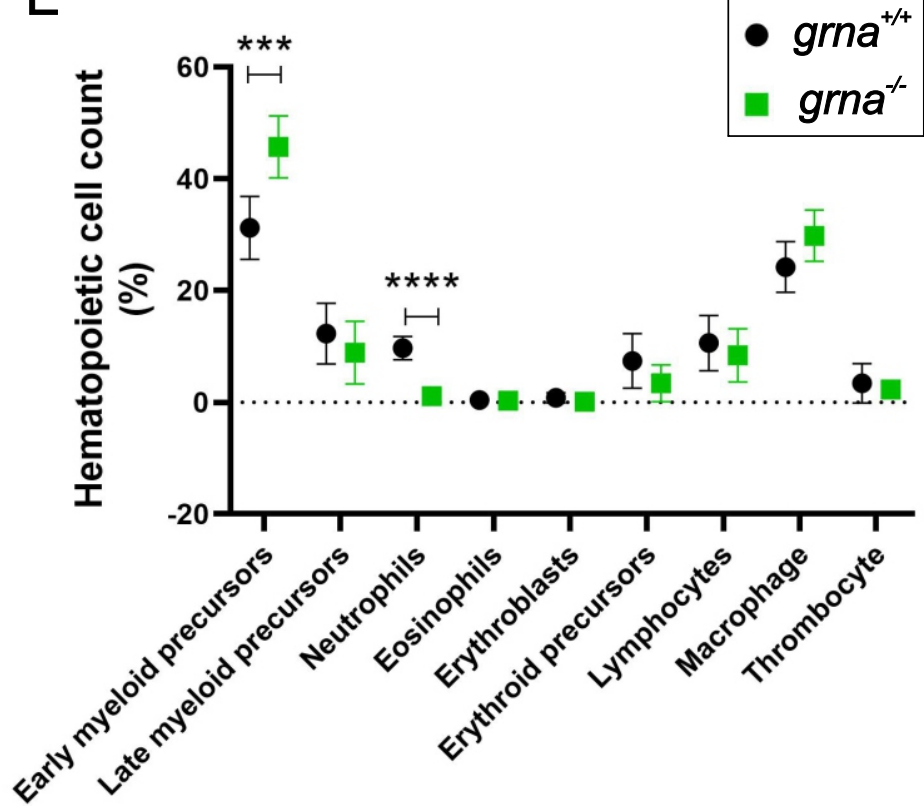
A

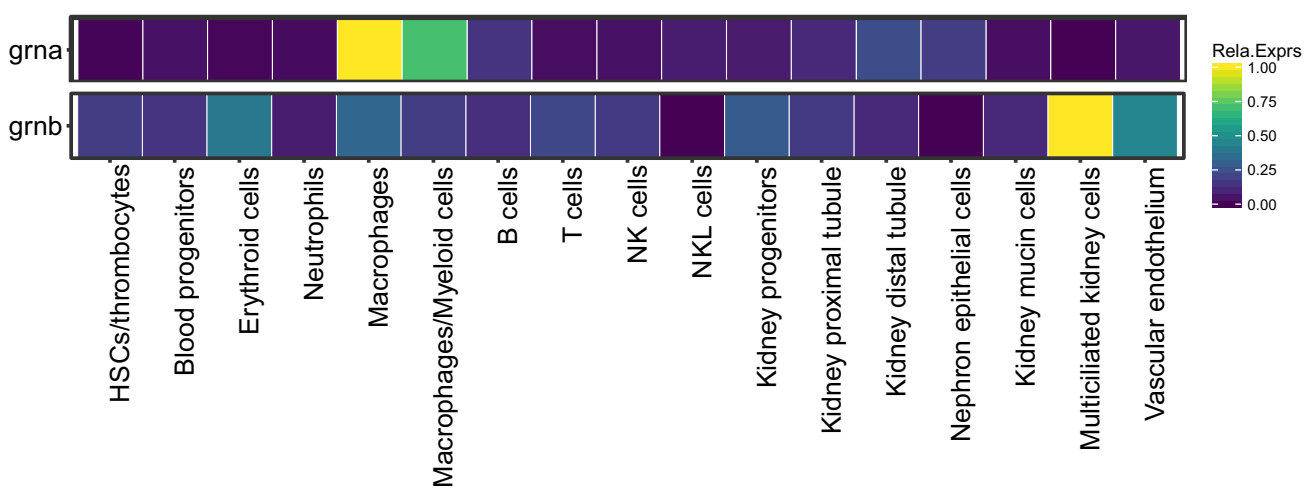

B \begin{tabular}{lllll} 
HSCP-1 & HSCP-2 & Meg Ery Multi MDP & Mono & Gran Myelo \\
\hline LSK $\odot \mathrm{CMP} \bullet \mathrm{GMP}$ & OLK CD34+
\end{tabular}

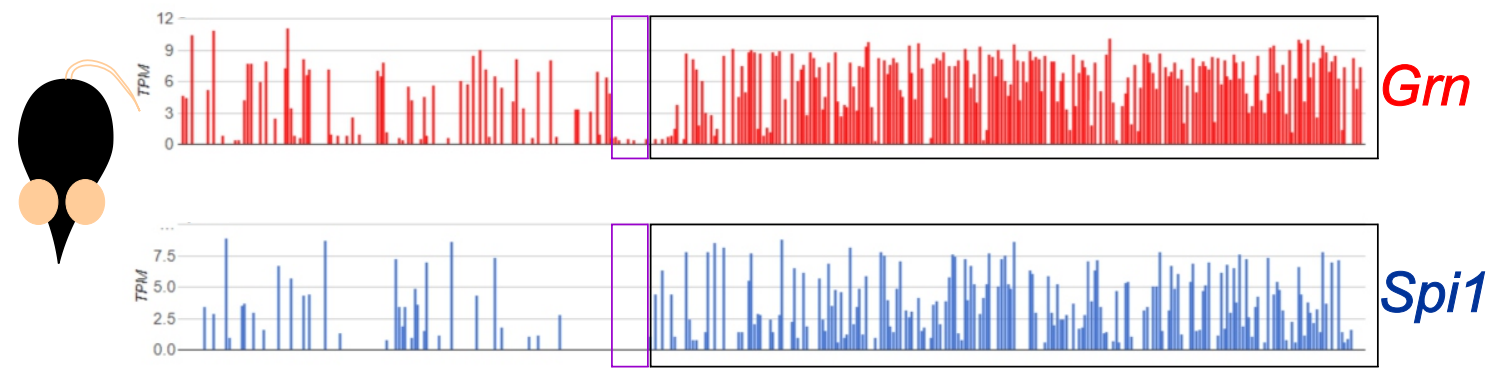

C

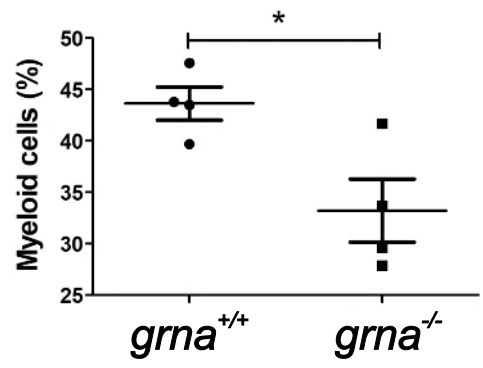

D

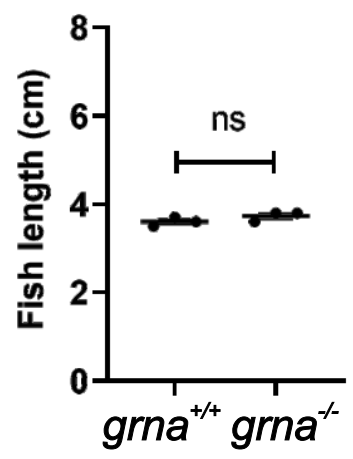


A Down-regulated
genes in grrna $\quad 154\left(11,595 \quad 116 \begin{array}{c}\text { Up-regulated } \\ \text { genes in grna-1 }\end{array}\right.$

B

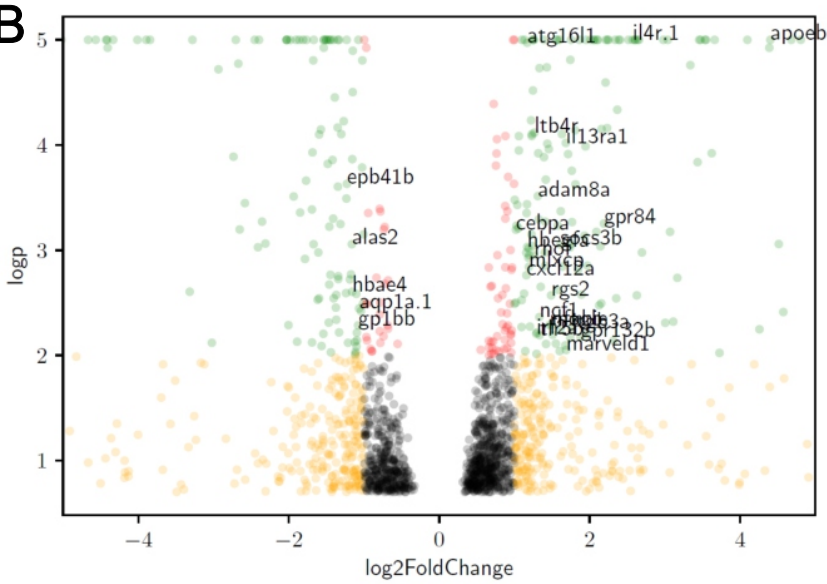

\section{Control}
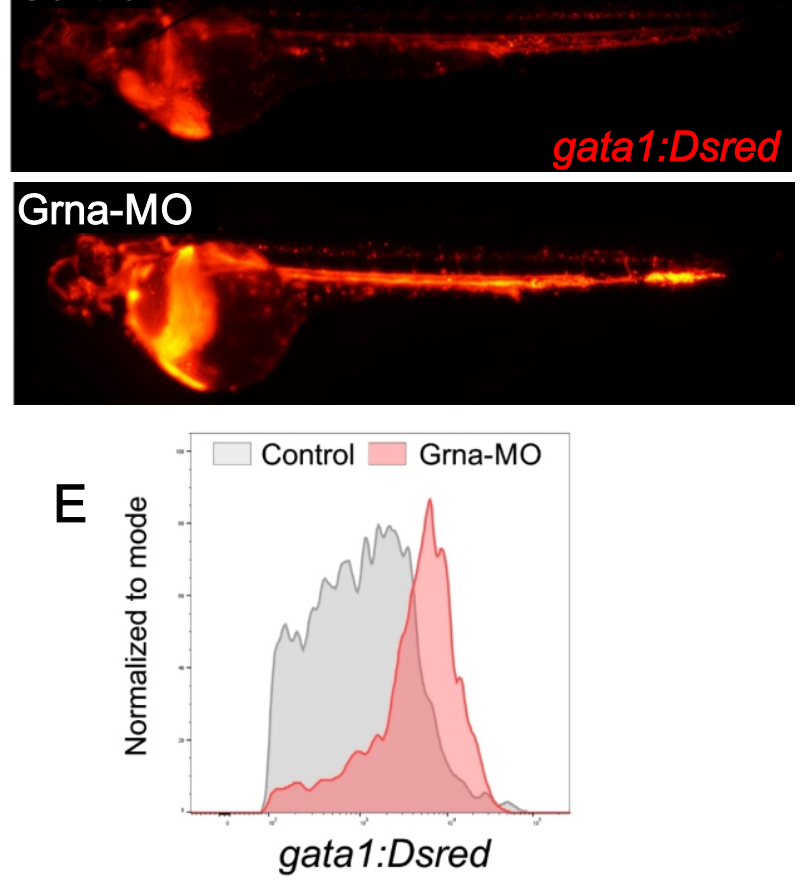

C

Figure 4

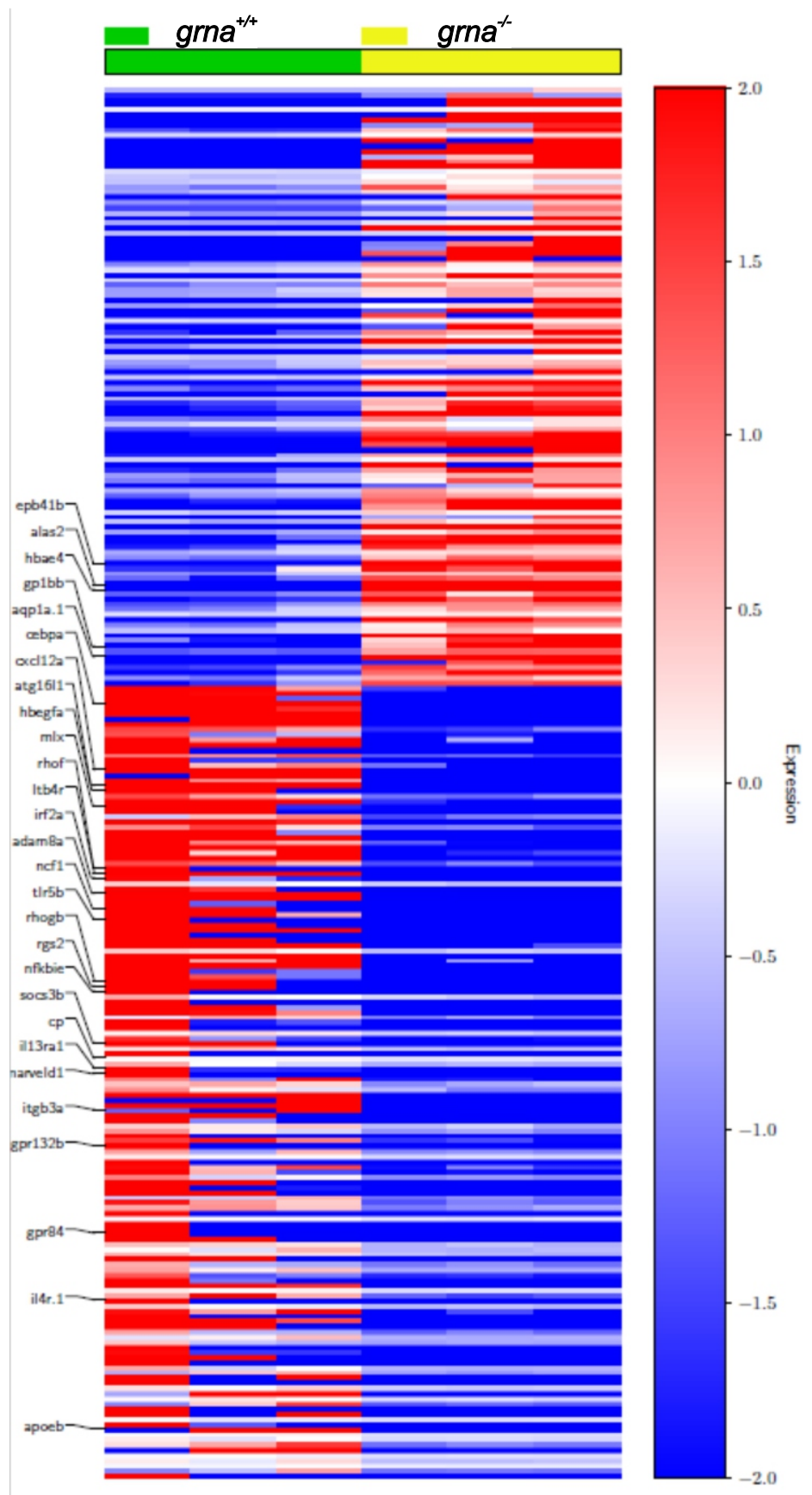


Sup Figure 4

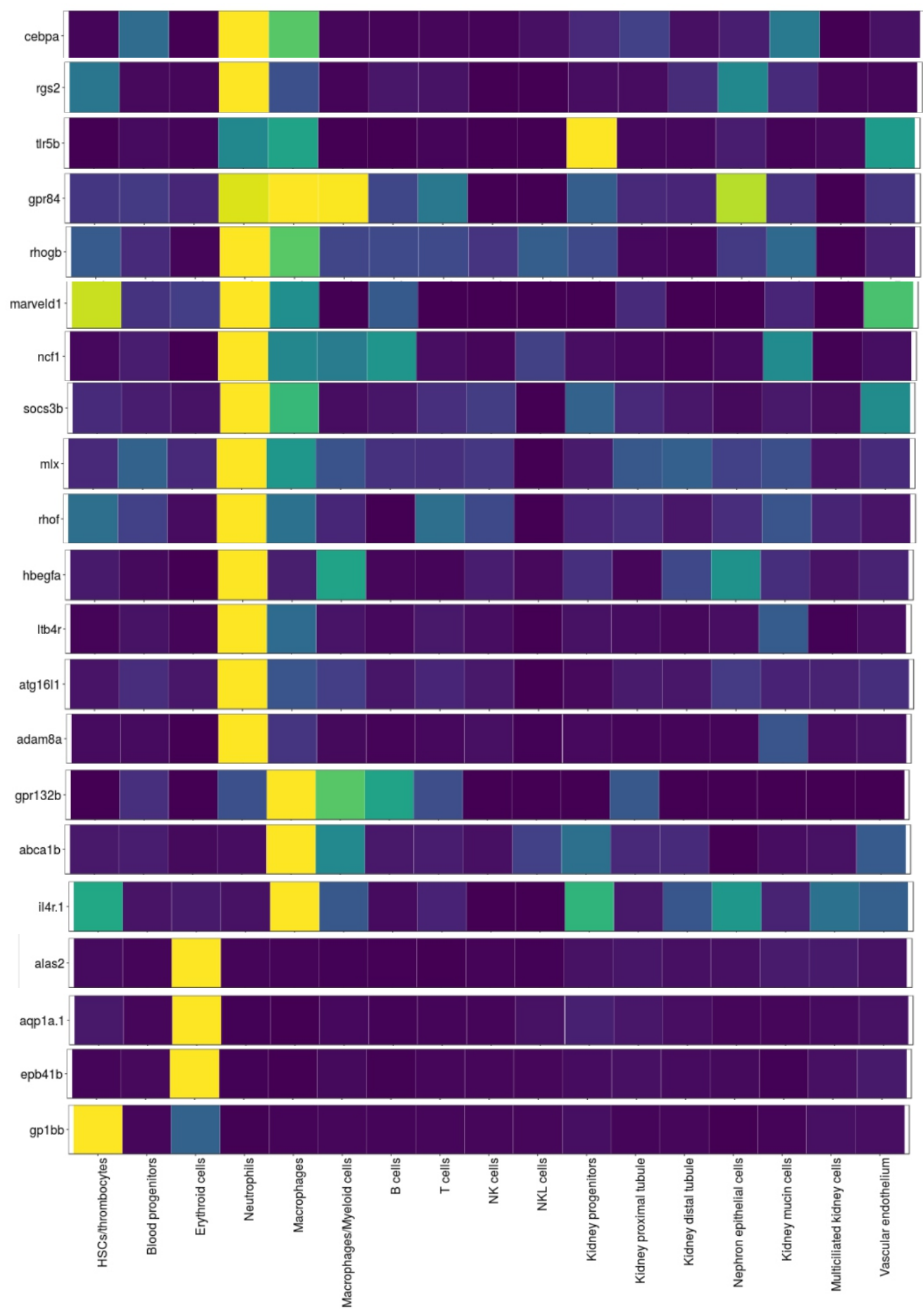

Rela.Exprs

1.00

0.75

0.50

0.25 
Figure 5

A

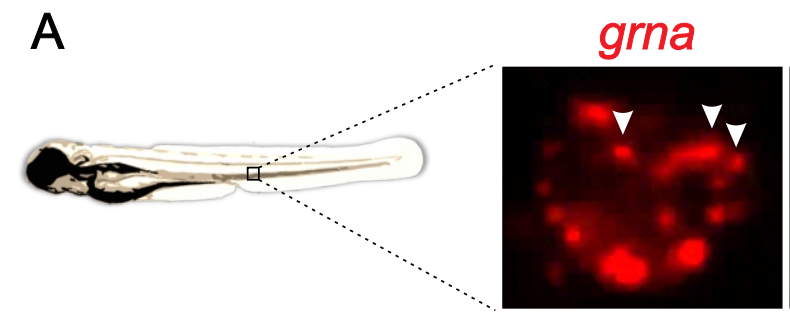

pu.1
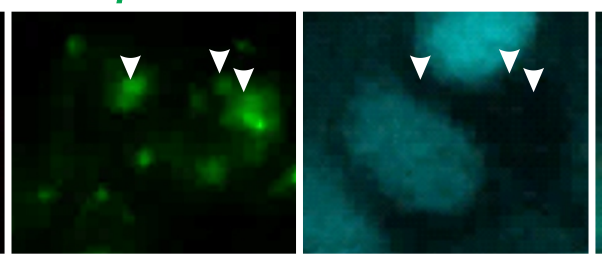

Merge

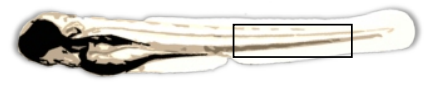

B

$$
\text { Pu.1-MO }
$$

irf8-MO

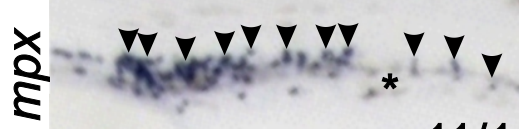

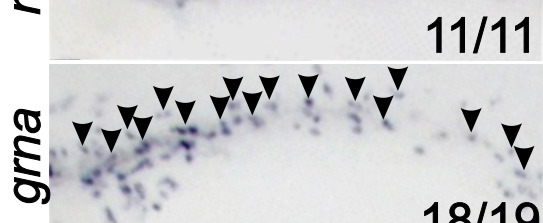

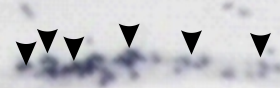

$11 / 11$

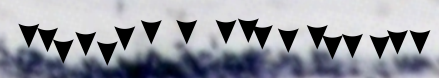

$8 / 8$

C

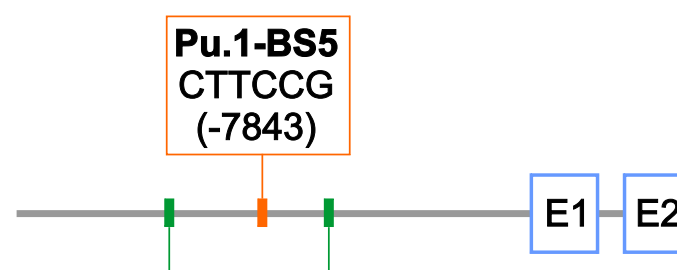

Pu.1-BS3

AAAAAAGGGAAAGTT $(-14045)$

\section{Pu.1-BS2}

CAAAAGAGGAAAGA $(-6935)$

D

pU.1-target site

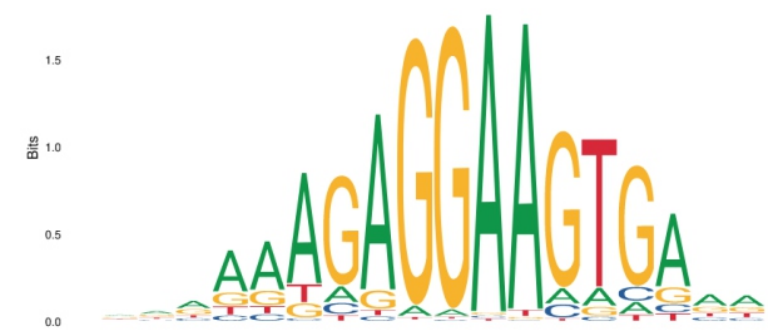

Pu.1-BS6

CTTCCG

(+4485)

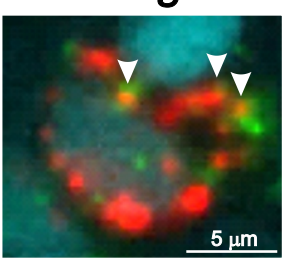

$5 \mu \mathrm{m}$

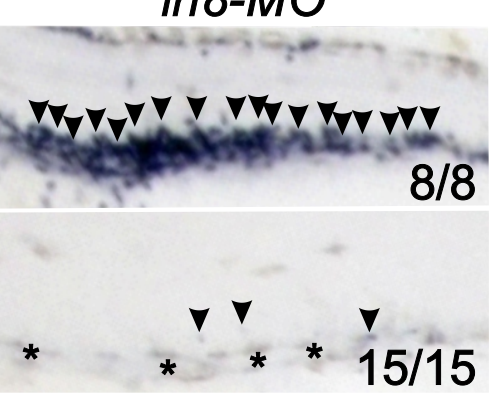


A

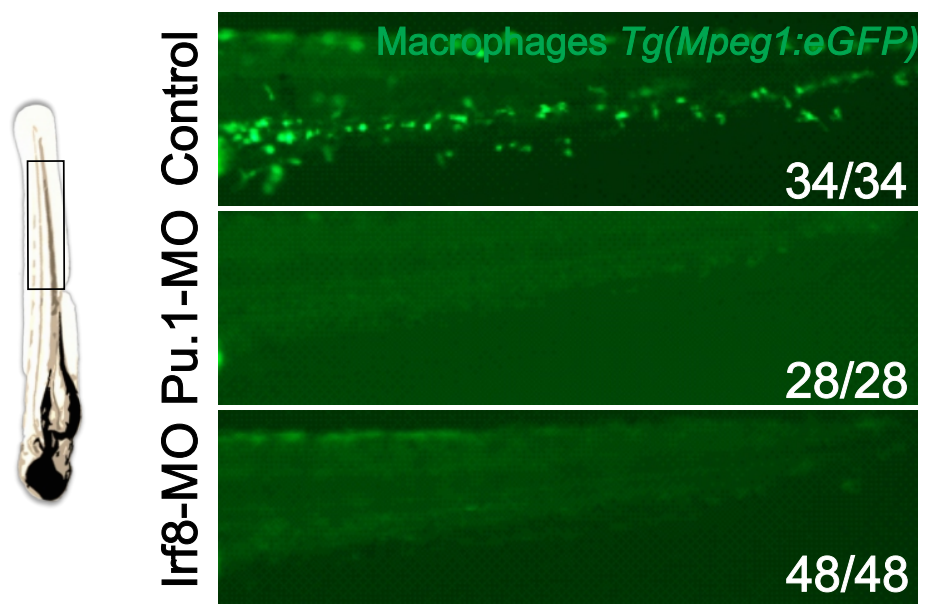

C

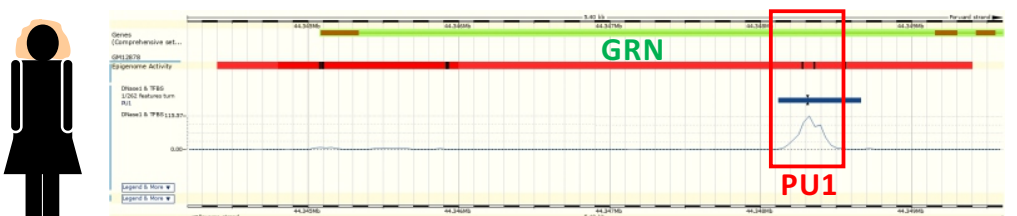

GRN promoter
B

Probeset Meta Profile 11939 microarrays in GEO analyzed

Signal Gene Intensity Expression

[log2] Activity

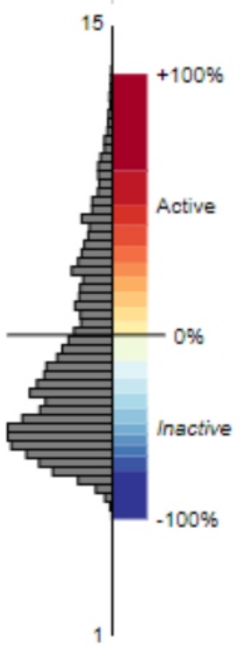

8.81

Dynamic-range [log2]
Sup Figure 5

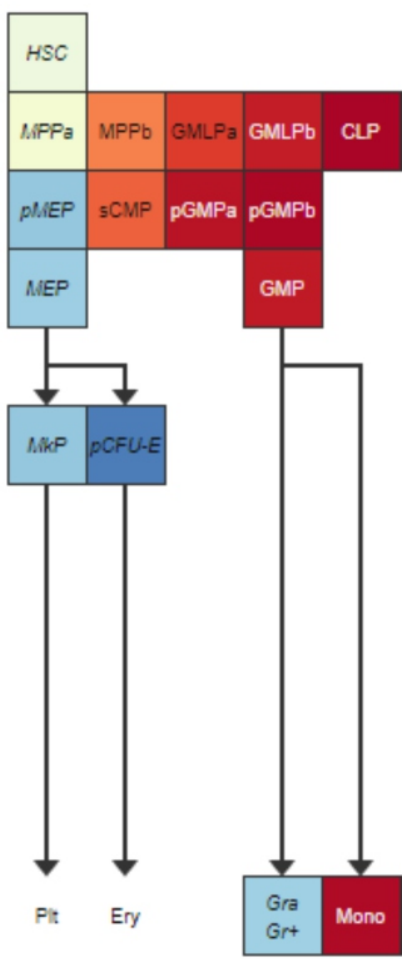

D Transcription factors that bind $G R N$ promoter

IRF8 21731497 ChIP-ChIP J774 Mouse

CEBPB 23403033 ChIP-Seq LIVER Mouse

NFE2L2 22581777 ChIP-Seq LYMPHOBLASTOID Human

PDX1 19855005 ChIP-ChIP MIN6 Mouse

TFEB 21752829 ChIP-Seq HELA Human

NANOG 21062744 ChIP-ChIP HESCS Human

TBP 23326641 ChIP-Seq C3H10T1-2 Mouse

RUNX1 20887958 ChIP-Seq HPC-7 Mouse

RELA 24523406 ChIP-Seq FIBROSARCOMA Human

GF1 26923725 Chip-Seq HPCs Mouse

E Transcription factors co-expressed with $G R N$ IRF8 27001747 Chip-Seq BMDM Mouse

SMRT 22465074 ChIP-Seq MACROPHAGES Mouse
SPI1 23547873 ChIP-Seq NB4 Human
Nerf2 26677805 Chip-Seq MACROPHAGESS Mouse
NCOR 22465074 ChIP-Seq MACROPHAGES Mouse
IRF8 21731497 ChIP-ChIP J774 Mouse
MECOM 23826213 hiP-Seq KASUMI Mouse
SPI1 22790984 ChIP-Seq ERYTHROLEUKEMIA Mouse
SPI1 22096565 ChIP-ChIP GC-B Mouse
BRD4 27068464 Chip-Seq AML-cells Mouse

F Genes co-expressed with GRN

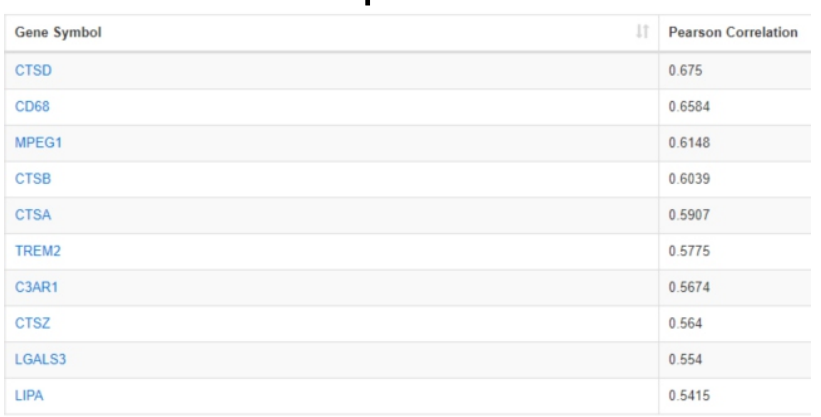


Figure 6

A

Morpholino injection

Grna-MO

Grna Mismatch MO

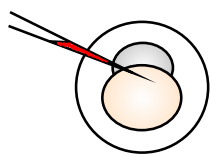

$\rightarrow$ COS $\rightarrow$

Tail resection

Tg(Mpeg1:eGFP)

Hours post fertilization

Hours

post wounding

\begin{tabular}{cccc}
0 hpf & $\mathbf{4 8 h p f}$ & $\mathbf{4 9} \mathbf{h p f}$ & $\mathbf{8 0 ~ h p f}$ \\
\hline $0 \mathrm{hpw}$ & $1 \mathrm{hpw}$ & $32 \mathrm{hpw}$
\end{tabular}

B
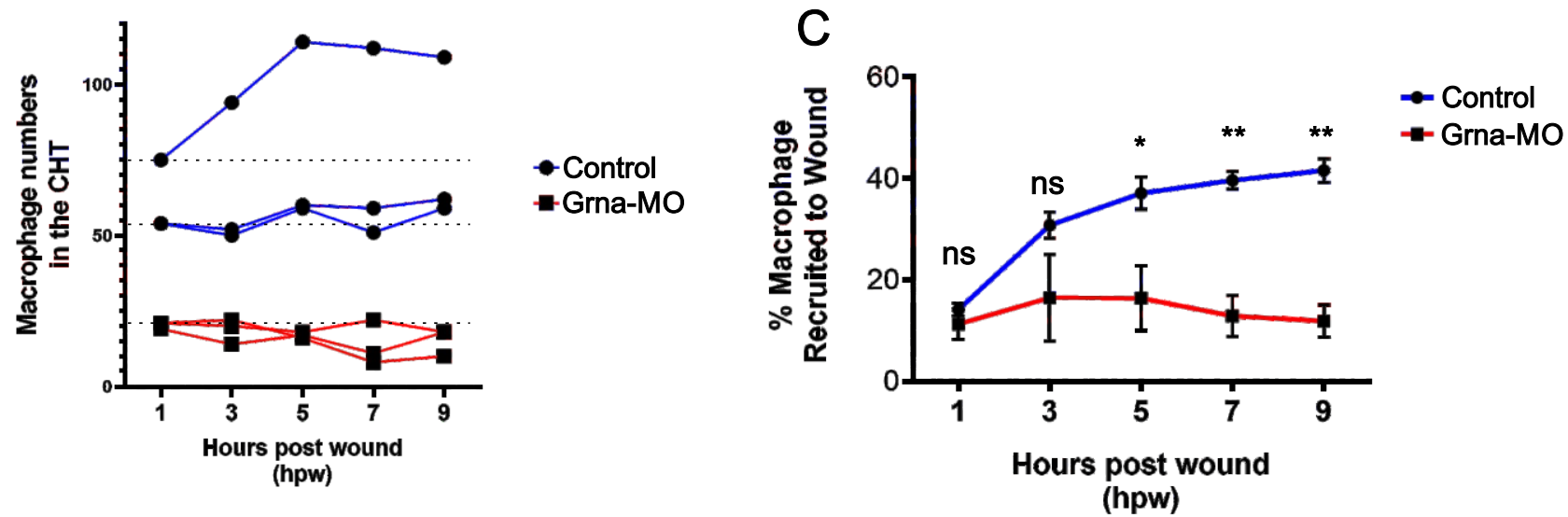

D

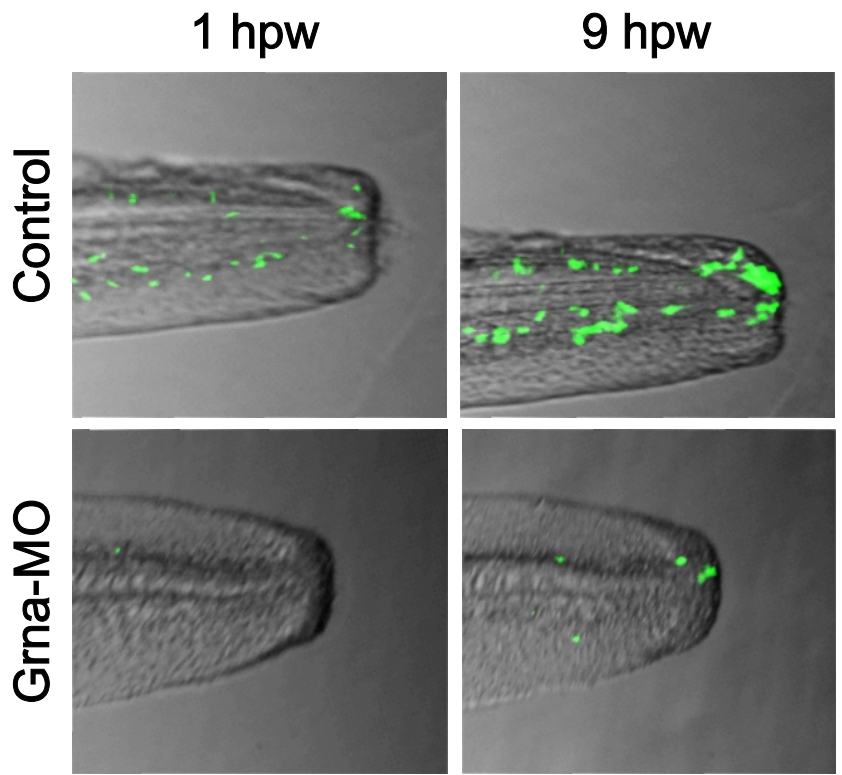
cence imaging of the tail

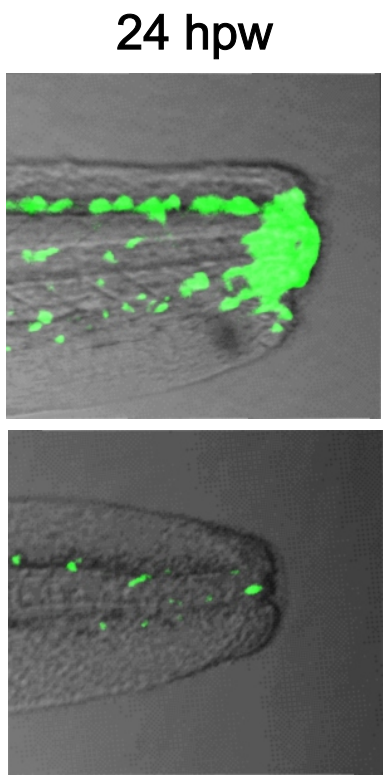

$32 \mathrm{hpw}$

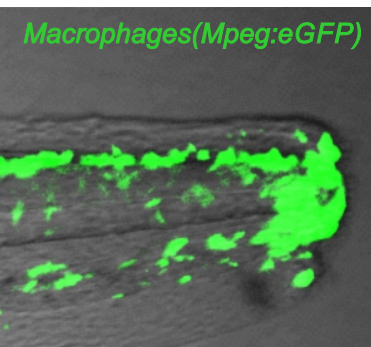

- Control

- Grna-MO

(hpw)

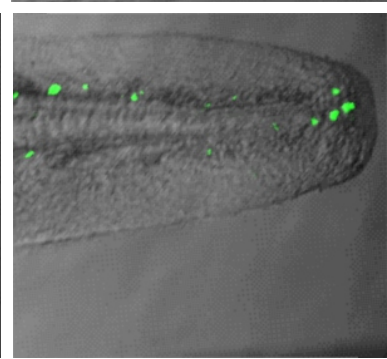


A

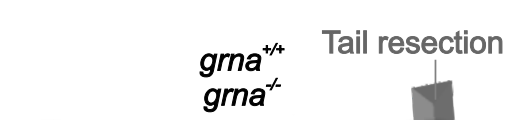

Figure 7

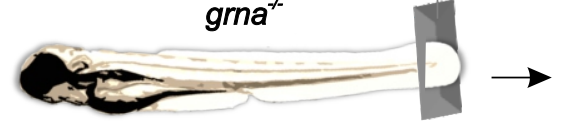

Hours

post fertilization

Hours

post wounding

B Ohpw

24hpw

Imaging

Tail fin regeneration
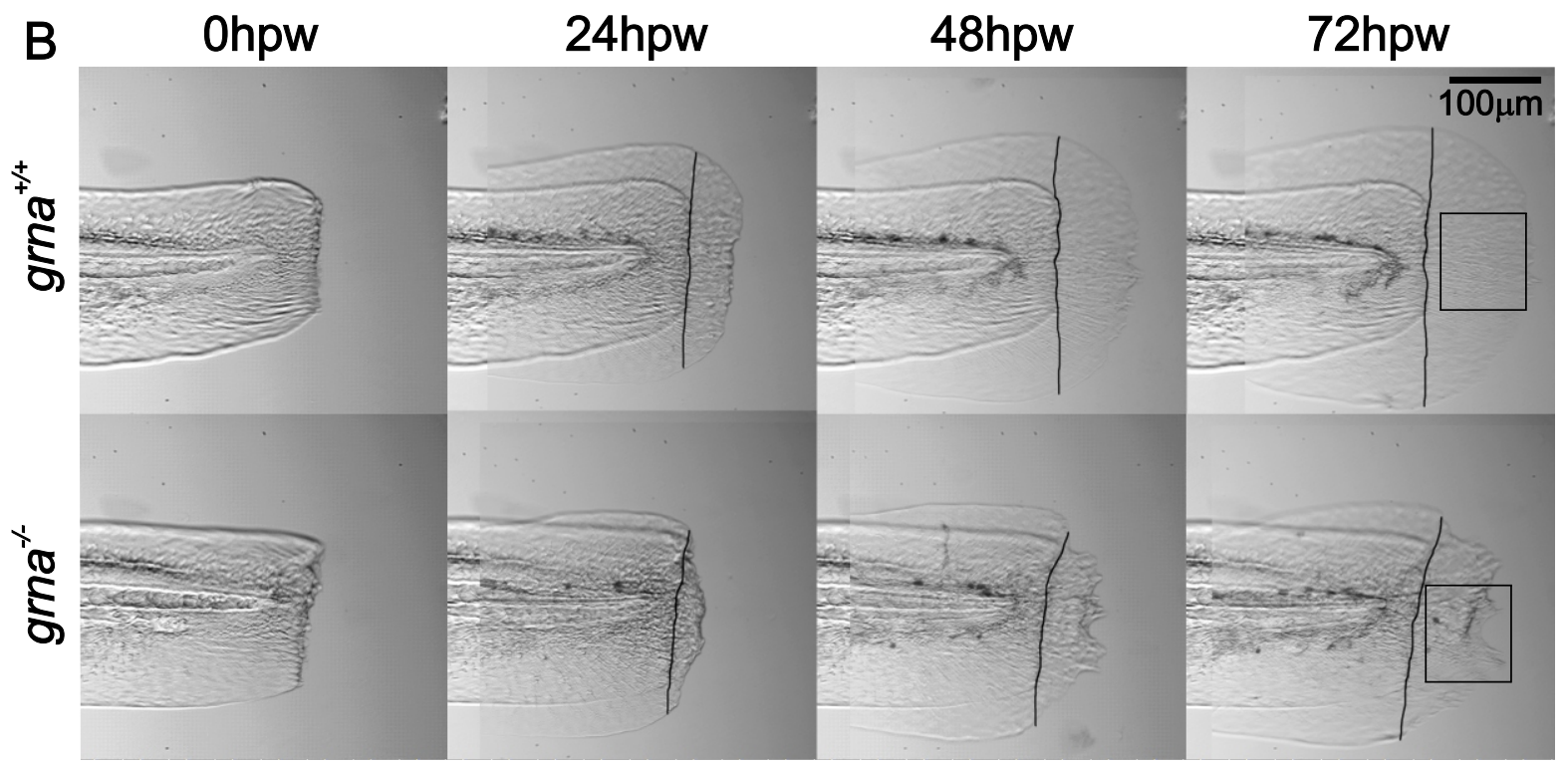

C

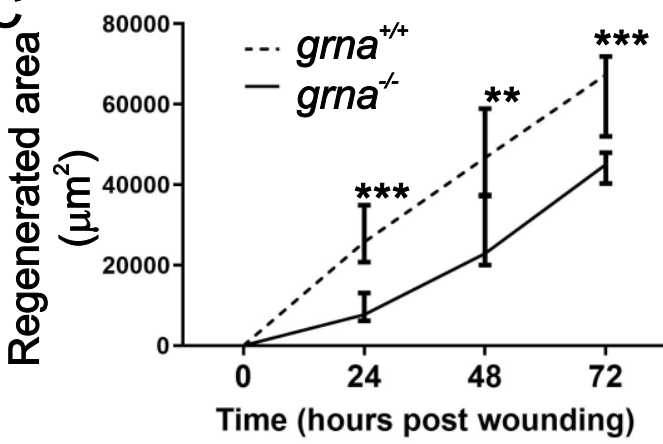

D

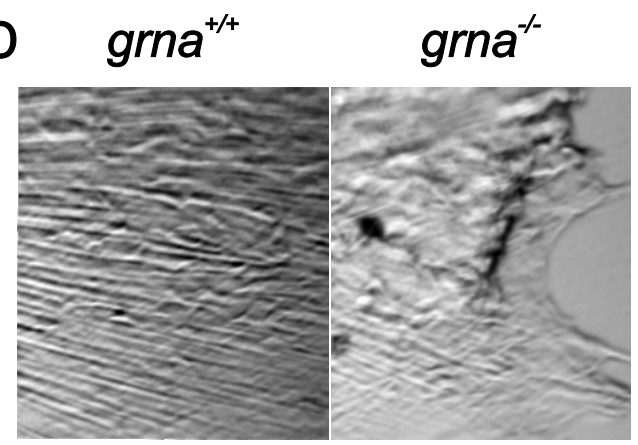

$E$

Myeloid progenitor

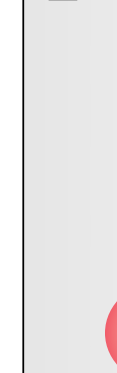

Alas2, Epb41b

Hbae4

Erythrocyte

Gata1

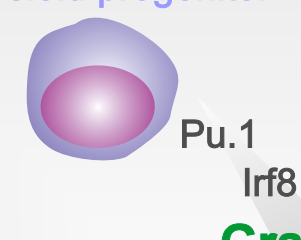

Granulin

Rgs2

Cebpa

$120 \mathrm{hpf}$

72 hpw

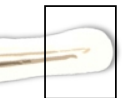


bioRxiv preprint doi: https://doi.org/10.1101/2020.07.23.217067; this version posted July 23, 2020. The copyright holder for this preprint (which was not certified by peer review) is the author/funder. All rights reserved. No reuse allowed without permission.

Table S1: Primers used in this manuscript.

\begin{tabular}{|c|c|c|c|c|}
\hline $\begin{array}{l}\text { Gene or } \\
\text { locus }\end{array}$ & Accession & Name & Nucleotide sequence $\left(5^{\prime} \rightarrow 3^{\prime}\right)$ & Use \\
\hline \multirow{2}{*}{ ef1a } & \multirow{2}{*}{ NM_131263.1 } & $\mathrm{F}$ & GAGAAGTTCGAGAAGGAAGC & \multirow{6}{*}{$q-P C R$} \\
\hline & & $\mathrm{R}$ & CGTAGTATTTGCTGGTCTCG & \\
\hline \multirow{2}{*}{ grna } & \multirow{2}{*}{$\begin{array}{c}\text { NM_00100194 } \\
9.3\end{array}$} & $\mathrm{~F}$ & AGCCAGACCTTCCCAAATCAT & \\
\hline & & $\mathrm{R}$ & CTCAGCAGGACAGGAAGAGC & \\
\hline \multirow{2}{*}{$g r n b$} & \multirow{2}{*}{ NM_212738.1 } & $\mathrm{F}$ & CGGCAAGAGTCTGGAAGAGT & \\
\hline & & $\mathrm{R}$ & CACACGGCCTTGACTAGAGG & \\
\hline \multirow{2}{*}{ grnb } & \multirow{2}{*}{ NM_130944.1 } & $\mathrm{F} 2-\mathrm{MO}$ & AACTTTGTGCGACCTGGAAC & \multirow{2}{*}{$\begin{array}{l}\text { Morpholino } \\
\text { validation }\end{array}$} \\
\hline & & $\mathrm{R} 2-\mathrm{MO}$ & TCTGGACAGCAGTGCTTTTG & \\
\hline \multirow{2}{*}{ grna } & \multirow{2}{*}{ NC_007114.7 } & pU.1-BS1-F & CACGAAAAGCCGAACTCT & \multirow{12}{*}{$\begin{array}{c}\text { CUT\&RUN } \\
\text {-qPCR }\end{array}$} \\
\hline & & pU.1-BS1-R & GTTGGTCAGGCCTTTGAC & \\
\hline \multirow{2}{*}{$\begin{array}{c}\text { grna 5' } \\
\text { enhancer }\end{array}$} & \multirow{2}{*}{ NC_007114.7 } & pU.1-BS2-F & GCAGACAAAAGAGGAAAGAAGC & \\
\hline & & pU.1-BS2-R & ACAGAACAGATCCTGATG & \\
\hline \multirow{2}{*}{$\begin{array}{c}\text { grna } 5 \\
\text { enhancer }\end{array}$} & \multirow{2}{*}{ NC_007114.7 } & pU.1-BS3-F & GCAGATGATGATGTAAAAAAGGG & \\
\hline & & pU.1-BS3-R & CACATTTCСTTTTACTTAACGC & \\
\hline \multirow{2}{*}{ grna } & \multirow{2}{*}{ NC_007114.7 } & Control-F & TGTCTTCTGATCTCAGTTCT & \\
\hline & & Control-R & GAGATAGACACGTACAGTTTG & \\
\hline \multirow{2}{*}{$\begin{array}{l}\text { grna 5' } \\
\text { enhancer }\end{array}$} & \multirow{2}{*}{ NC_007114.7 } & pU.1-BS5-F & GTTTCACGTAGCGGAAGC & \\
\hline & & pU.1-BS5-R & GGGTTGTGGCTGAAAAGG & \\
\hline \multirow[b]{2}{*}{ grna } & \multirow[b]{2}{*}{ NC_007114.7 } & pU.1-BS6-F & GCGAGTTTGAGCCACTTG & \\
\hline & & pU.1-BS6-R & CCTGATCACTTCCGGGAT & \\
\hline
\end{tabular}

